\title{
ESTIMATES FOR EXPONENTIAL SUMS WITH A LARGE AUTOMORPHISM GROUP
}

\author{
ANTONIO ROJAS-LEÓN
}

\begin{abstract}
We prove some improvements of the classical Weil bound for one variable additive and multiplicative character sums associated to a polynomial over a finite field $k=\mathbb{F}_{q}$ for two classes of polynomials which are invariant under a large abelian group of automorphisms of the affine line $\mathbb{A}_{k}^{1}$ : those invariant under translation by elements of $k$ and those invariant under homotheties with ratios in a large subgroup of the multiplicative group of $k$. In both cases, we are able to improve the bound by a factor of $\sqrt{q}$ over an extension of $k$ of cardinality sufficiently large compared to the degree of $f$.
\end{abstract}

\section{INTRODUCTION}

Let $k=\mathbb{F}_{q}$ be a finite field with $q$ elements. As a consequence Weil's bound for the number of rational points on a curve over $k$, one can obtain estimates for character sums defined on the affine line $\mathbb{A}_{k}^{1}$ (cf. [6], [17]). Let us describe the precise results.

Let $f \in k[x]$ be a polynomial of degree $d$ and $\psi: k \rightarrow \mathbb{C}^{\star}$ a non-trivial additive character. Consider the sum $\sum_{x \in k} \psi(f(x))$ (and, more generally, $\sum_{x \in k_{r}} \psi\left(\operatorname{Tr}_{k_{r} / k}(f(x))\right.$ ) for a finite extension $k_{r}$ of $k$ of degree $r$ ). Then, if $d$ is prime to $p$, we have the estimate

$$
\left|\sum_{x \in k_{r}} \psi\left(\operatorname{Tr}_{k_{r} / k}(f(x))\right)\right| \leq(d-1) q^{\frac{r}{2}} .
$$

If $d$ is divisible by $p$, we can reduce to the previous case using the following trick. Since $t \mapsto \psi\left(t^{p}\right)$ is a non-trivial additive character, there must be some $a \in k$ such that $\psi\left(t^{p}\right)=\psi(a t)$ for every $t \in k$. If $f(x)=a_{d} x^{d}+a_{d-1} x^{d-1}+\cdots$ with $d=e p$, let $b_{d} \in k$ be such that $b_{d}^{p}=a_{d}$, then

$$
\begin{gathered}
\psi\left(\operatorname{Tr}_{k_{r} / k}(f(x))\right)=\psi\left(\operatorname{Tr}_{k_{r} / k}\left(\left(b_{d} x^{e}\right)^{p}\right)\right) \psi\left(\operatorname{Tr}_{k_{r} / k}\left(f(x)-a_{d} x^{d}\right)\right)= \\
=\psi\left(\operatorname{Tr}_{k_{r} / k}\left(b_{d} x^{e}\right)^{p}\right) \psi\left(\operatorname{Tr}_{k_{r} / k}\left(f(x)-a_{d} x^{d}\right)\right)=\psi\left(a \cdot \operatorname{Tr}_{k_{r} / k}\left(b_{d} x^{e}\right)\right) \psi\left(\operatorname{Tr}_{k_{r} / k}\left(f(x)-a_{d} x^{d}\right)\right)= \\
=\psi\left(\operatorname{Tr}_{k_{r} / k}\left(f(x)-a_{d} x^{d}+a b_{d} x^{e}\right)\right) .
\end{gathered}
$$

We keep reducing the polynomial in this way until we get a polynomial with degree $d^{\prime}$ prime to $p$. Then we apply the prime to $p$ case and obtain an estimate

$$
\left|\sum_{x \in k_{r}} \psi\left(\operatorname{Tr}_{k_{r} / k}(f(x))\right)\right| \leq\left(d^{\prime}-1\right) q^{\frac{r}{2}} .
$$

except when $d^{\prime}$ is zero (that is, when $f=c+g^{p}-a g$ for some constant $c$ and sone $g \in k[x])$. If the character $\psi$ is obtained from a character of the prime subfield $\mathbb{F}_{p}$ by pulling back via the trace map, then $a=1$.

Partially supported by P08-FQM-03894 (Junta de Andalucía), MTM2007-66929 and FEDER. 
Similarly, if $\chi: k^{\star} \rightarrow \mathbb{C}^{\star}$ is a multiplicative character of order $m>1$ and $f \in k[x]$ is not an $m$-th power, we have an estimate

$$
\left|\sum_{x \in k_{r}} \chi\left(\mathrm{N}_{k_{r} / k}(f(x))\right)\right| \leq(e-1) q^{\frac{r}{2}} \leq(d-1) q^{\frac{r}{2}}
$$

where $e$ is the number of distinct roots of $f$.

In this article we will improve these estimates for a special class of polynomials: those which are either translation invariant or homothety invariant, that is, either $f(x+\lambda)=f(x)$ for every $\lambda \in k$ or $f(\lambda x)=f(x)$ for every $\lambda \in k^{\star}$ (or every $\lambda$ in a large subgroup of $k^{\star}$ ). For such polynomials, there is a large abelian group $G$ of automorphisms of $\mathbb{A}_{k}^{1}$ such that $f \circ \sigma=f$ for every $\sigma \in G$.

On the level of $\ell$-adic cohomology, this gives an action of $G$ on the pull-back by $f$ of the Artin-Schreier and Kummer sheaves associated to $\psi$ and $\chi$ respectively [1. 1.7], so they induce an action on their cohomology. The character sums can be expressed as the trace of the geometric $k_{r}$-Frobenius action on this cohomology, by Grothendieck's trace formula. The above estimates are a consequence of the fact that this action has all eigenvalues of archimedean absolute value $\leq q^{\frac{r}{2}}$. Precisely, if $S_{r}=\sum_{x \in k_{r}} \psi\left(\operatorname{Tr}_{k_{r} / k}(f(x))\right)$ (respectively $U_{r}=\sum_{x \in k_{r}} \chi\left(\mathrm{N}_{k_{r} / k}(f(x))\right)$ ) the $L$ functions

$$
L(\psi, f ; T):=\exp \sum_{r \geq 1} S_{r} \frac{T^{r}}{r}
$$

and

$$
L(\chi, f ; T):=\exp \sum_{r \geq 1} U_{r} \frac{T^{r}}{r}
$$

are the polynomials $\operatorname{det}\left(1-T \cdot \operatorname{Frob}_{k} \mid \mathrm{H}_{c}^{1}\left(\mathbb{A}_{\bar{k}}, f^{\star} \mathcal{L}_{\psi}\right)\right)$ and $\operatorname{det}\left(1-T \cdot \operatorname{Frob}_{k} \mid \mathrm{H}_{c}^{1}\left(\mathbb{A} \frac{1}{k}, f^{\star} \mathcal{L}_{\chi}\right)\right)$, of degree $d^{\prime}-1$ and $e-1$ respectively.

Now under the action of the abelian group $G$, this cohomology splits as a direct sum of eigenspaces for the different characters of $G$. Under certain generic conditions, it is natural to expect some cancellation among the traces of the Frobenius actions on these eigenspaces, thus giving a substantial improvement of Weil's estimate if $G$ is large (namely by a $\sqrt{\# G}$ factor). Compare [15], where an improvement for the Weil estimate for the number of rational points on Artin-Schreier curves was obtained using the same arguments we apply in this article.

For the translation invariant case (sections 2 ans 3), we obtain this improvement using the local theory of $\ell$-adic Fourier transform [14] and Katz' computation of the geometric monodromy groups for some families of exponential sums [7], [9]. The argument is similar to that in [15. For the homothety invariant case (sections 4 and 5), we use Weil descent together with certain properties of the convolution of sheaves on $\mathbb{G}_{m, k}$.

Throughout this article, $k=\mathbb{F}_{q}$ will be a finite field of characteristic $p, \bar{k}=\overline{\mathbb{F}}_{q}$ a fixed algebraic closure and $k_{r}=\mathbb{F}_{q^{r}}$ the unique extension of $k$ of degree $r$ in $\bar{k}$. We will fix a prime $\ell \neq p$, and work with $\ell$-adic cohomology. In order to speak about weights without ambiguity, we will fix a field isomorphism $\iota: \overline{\mathbb{Q}}_{\ell} \rightarrow \mathbb{C}$. We will use this isomorphism to identify $\overline{\mathbb{Q}}_{\ell}$ and $\mathbb{C}$ without making any further mention to it. When we speak about weights, we will mean weights with respect to the chosen isomorphism $\iota$. 


\section{AdDitive CHARACTER SUMS FOR TRANSLATION INVARIANT POLYNOMIALS}

Let $f \in k[x]$ be a polynomial. $f$ is said to be translation invariant if $f(x+a)=$ $f(x)$ for every $a \in k$.

Lemma 2.1. Let $f \in k[x]$. The following conditions are equivalent:

(a) $f$ is translation invariant.

(b) There exists $g \in k[x]$ such that $f(x)=g\left(x^{q}-x\right)$.

Proof. $(b) \Rightarrow(a)$ is clear. Suppose that $f$ is translation invariant. If the degree of $f$ is $<q$, the polynomial $f(x)-f(0)$ has at least $q$ roots (all elements of $k$ ) and degree $<q$, so it is identically zero. So $f$ is the constant polynomial $f(0)$. Otherwise, we can write $f(x)=\left(x^{q}-x\right) h(x)+r(x)$ with $\operatorname{deg}(r)<q$. For every $a \in k$ we have then $f(x+a)=\left(x^{q}-x\right) h(x+a)+r(x+a)=\left(x^{q}-x\right) h(x)+r(x)$, so $\left(x^{q}-x\right)(h(x+a)-h(x))=r(x)-r(x+a)$. Since the right hand side has degree $<q$, we conclude that $h(x+a)-h(x)=r(x+a)-r(x)=0 . r(x)$ is then translation invariant and therefore constant, for its degree is less than $q$, and $h$ is also translation invariant of degree $\operatorname{deg}(f)-q$. By induction, there is $t \in k[x]$ such that $h(x)=t\left(x^{q}-x\right)$. So we take $g(x)=x t(x)+r$.

Let $f \in k[x]$ be translation invariant, and $g \in k[x]$ of degree $d$ such that $f(x)=g\left(x^{q}-x\right)$. Let $\psi: k \rightarrow \overline{\mathbb{Q}}_{\ell}^{\star}$ be a non-trivial additive character. The ArtinScheier-reduced degree of $f$ (i.e. the lowest degree of a polynomial which is ArtinSchreier equivalent to $f)$ is $q(d-1)+1\left(\right.$ since $g\left(x^{q}-x\right)=a_{d} x^{q d}+d a_{d} x^{q(d-1)+1}+$ (terms of degree $\leq q(d-1))$ ). Therefore the Weil bound for exponential sums gives

$$
\left|\sum_{x \in k_{r}} \psi\left(\operatorname{Tr}_{k_{r} / k}(f(x))\right)\right| \leq q(d-1) q^{\frac{r}{2}}=(d-1) q^{\frac{r}{2}+1}
$$

On the other hand, since $f(x)=g\left(x^{q}-x\right)$ we get, for every $r \geq 1$,

$$
\begin{gathered}
\sum_{x \in k_{r}} \psi\left(\operatorname{Tr}_{k_{r} / k}(f(x))\right)=\sum_{x \in k_{r}} \psi\left(\operatorname{Tr}_{k_{r} / k}\left(g\left(x^{q}-x\right)\right)\right)= \\
=\sum_{t \in k_{r}} \#\left\{x \in k_{r} \mid x^{q}-x=t\right\} \psi\left(\operatorname{Tr}_{k_{r} / k}(g(t))\right)= \\
=\sum_{t \in k_{r}} \sum_{u \in k} \psi\left(u \operatorname{Tr}_{k_{r} / k}(t)\right) \psi\left(\operatorname{Tr}_{k_{r} / k}(g(t))\right)=\sum_{u \in k} \sum_{t \in k_{r}} \psi\left(\operatorname{Tr}_{k_{r} / k}(g(t)+u t)\right) .
\end{gathered}
$$

Consider the $\overline{\mathbb{Q}}_{\ell}$-sheaf $\mathcal{L}_{\psi(g)}:=g^{\star} \mathcal{L}_{\psi}$ on $\mathbb{A}_{k}^{1}$, where $\mathcal{L}_{\psi}$ is the Artin-Schreier sheaf associated to $\psi$. The Fourier transform of the object $\mathcal{L}_{\psi(g)}$ [1] with respect to $\psi$ [13] is a single sheaf $\mathcal{F}_{g}$ placed in degree -1 . The sheaf $\mathcal{F}_{g}$ is irreducible and smooth of rank $d-1$ on $\mathbb{A}_{k}^{1}$, and totally wild at infinity with a single slope $\frac{d}{d-1}$ and Swan conductor $d$ [7, Theorem 17]. We have

$$
\begin{gathered}
\sum_{x \in k_{r}} \psi\left(\operatorname{Tr}_{k_{r} / k}(f(x))\right)=\sum_{x \in k_{r}} \psi\left(\operatorname{Tr}_{k_{r} / k}\left(g\left(x^{q}-x\right)\right)\right)=\sum_{u \in k} \sum_{t \in k_{r}} \psi\left(\operatorname{Tr}_{k_{r} / k}(g(t)+u t)\right)= \\
\quad=-\sum_{u \in k} \operatorname{Tr}\left(\operatorname{Frob}_{k, u}^{r} \mid\left(\mathcal{F}_{g}\right)_{u}\right)=-\sum_{u \in k} \operatorname{Tr}\left(\operatorname{Frob}_{k, u} \mid\left[\mathcal{F}_{g}\right]_{u}^{r}\right)
\end{gathered}
$$

where $\left[\mathcal{F}_{g}\right]^{r}$ is the $r$-th Adams power of $\mathcal{F}_{g}$ [4]. 
Let $g(x)=\sum_{i=0}^{d} a_{i} x^{i}$. Recall the following facts about the local and global monodromies of the sheaf $\mathcal{F}_{g}$ :

(1) Suppose that $p>d$ and $k$ contains all 2(d-1)-th roots of $-d a_{d}$. Let $u(t)=$ $\sum_{i \geq 0} r_{i} t^{1-i} \in t k\left[\left[t^{-1}\right]\right]$ be a power series such that $f^{\prime}(t)+u(t)^{d-1}=0$ and let $v(t)=\sum_{i>0} s_{i} t^{1-i}$ be the inverse image of $t$ under the automorphism $k\left(\left(t^{-1}\right)\right) \rightarrow k\left(\left(t^{-1}\right)\right)$ defined by $t^{-1} \mapsto u(t)^{-1}$ (cf. 3. Proposition 3.1]). Let $h(t)=\sum_{i=0}^{d} b_{i} t^{i}$ be the polynomial obtained from $f(v(t))+v(t) t^{d-1} \in$ $t^{d} k\left[\left[t^{-1}\right]\right]$ by removing the terms with negative exponent. Then, as a representation of the decomposition group $D_{\infty}$ at infinity, we have

$\mathcal{F}_{g} \cong[d-1]_{\star}\left(\mathcal{L}_{\psi(h(t))} \otimes \mathcal{L}_{\rho^{d}\left(s_{0} t\right)}\right) \otimes \rho\left(d(d-1) a_{d} / 2\right)^{\operatorname{deg}} \otimes g(\rho, \psi)^{\operatorname{deg}}$

where $\rho: k^{\star} \rightarrow \overline{\mathbb{Q}}_{\ell}^{\star}$ is the quadratic character, $g(\rho, \psi)=-\sum_{t \in k} \rho(t) \psi(t)$ the corresponding Gauss sum and $[d-1]_{\star}: \mathbb{G}_{m, k} \rightarrow \mathbb{G}_{m, k}$ the $(d-1)$-th power map [5, Equation 3]. Notice that $s_{0}^{d-1}=-1 / d a_{d}$.

(2) Suppose that $p>2$, and let $G \subseteq \mathrm{GL}(V)$ be the geometric monodromy group of $\mathcal{F}_{g}$, where $V$ is its stalk at a geometric generic point. Then by [16. Propositions 11.1 and 11.6], either $G$ is finite or $G_{0}$ (the unit connected component of $G)$ is $\operatorname{SL}(V)$ or $\operatorname{Sp}(V)$ in its standard representation. By [7, proof of Theorem 19], for $p>d$ the Sp case occurs if and only if $g(x+c)+d$ is odd for some $c, d \in k$. Moreover for $p>2 d-1 G$ is never finite by [7, Theorem 19]. See [5, Section 2] for some other criterions that rule out the finite monodromy case in the $p \leq 2 d-1$ case.

The determinant of $\mathcal{F}_{g}$ is computed over $\bar{k}$ in [7, Theorem 17]. In order to obtain a good estimate in the exceptional case below, we need to find its value over $k$.

Lemma 2.2. Suppose that $p>d$ and $k$ contains all $2(d-1)$-th roots of $-d a_{d}$. Then

$\operatorname{det} \mathcal{F}_{g} \cong \mathcal{L}_{\psi\left((d-1) b_{d-1} t+(d-1) b_{0}\right)} \otimes \rho^{d}(-1)^{\operatorname{deg}} \otimes \rho^{d-1}\left(d(d-1) a_{d} / 2\right)^{\operatorname{deg}} \otimes\left(g(\rho, \psi)^{d-1}\right)^{\operatorname{deg}}$

Proof. Note that the result is compatible with [7, Theorem 17], since $b_{d-1}=$ $a_{d-1} s_{0}^{d-1}=a_{d-1} / r_{0}^{d-1}=-a_{d-1} / d a_{d}$ as one can easily check.

Let $D_{\infty}^{d-1} \subseteq D_{\infty}$ be the closed subgroup of index $d-1$ which fixes $1 / t^{d-1}$. Since $k$ contains all $(d-1)$-th roots of unity, $D_{\infty}^{d-1}$ is normal in $D_{\infty}$ and the quotient $D_{\infty} / D_{\infty}^{d-1}$ is generated by $t \mapsto \zeta t$, where $\zeta \in k$ is a primitive $(d-1)$-th root of unity. Using the previous description of the representation of $D_{\infty}$ given by $\mathcal{F}_{g}$, we get an isomorphism of $D_{\infty}^{d-1}$-representations

so

$$
[d-1]^{\star} \mathcal{F}_{g} \cong
$$$$
\cong\left(\bigoplus_{i=0}^{d-2}\left(t \mapsto \zeta^{i} t\right)^{\star} \mathcal{L}_{\psi(h(t))} \otimes \mathcal{L}_{\rho^{d}\left(s_{0} t\right)}\right) \otimes \rho\left(d(d-1) a_{d} / 2\right)^{\operatorname{deg}} \otimes g(\rho, \psi)^{d e g} \cong
$$$$
\cong\left(\bigoplus_{i=0}^{d-2} \mathcal{L}_{\psi\left(h\left(\zeta^{i} t\right)\right)} \otimes \mathcal{L}_{\rho^{d}\left(s_{0} \zeta^{i} t\right)}\right) \otimes \rho\left(d(d-1) a_{d} / 2\right)^{\operatorname{deg}} \otimes g(\rho, \psi)^{\operatorname{deg}}
$$

$$
\begin{gathered}
{[d-1]^{\star} \operatorname{det} \mathcal{F}_{g} \cong \operatorname{det}[d-1]^{\star} \mathcal{F}_{g} \cong} \\
\cong\left(\bigotimes_{i=0}^{d-2} \mathcal{L}_{\psi\left(h\left(\zeta^{i} t\right)\right)} \otimes \mathcal{L}_{\rho^{d}\left(s_{0} \zeta^{i} t\right)}\right) \otimes \rho^{d-1}\left(d(d-1) a_{d} / 2\right)^{\operatorname{deg}} \otimes\left(g(\rho, \psi)^{d-1}\right)^{d e g} \cong
\end{gathered}
$$


ESTIMATES FOR EXPONENTIAL SUMS WITH A LARGE AUTOMORPHISM GROUP 5

$$
\cong \mathcal{L}_{\psi\left(\sum_{i=0}^{d-2} h\left(\zeta^{i} t\right)\right)} \otimes \mathcal{L}_{\rho^{d}\left(\prod_{i=0}^{d-2}\left(s_{0} \zeta^{i} t\right)\right)} \otimes \rho^{d-1}\left(d(d-1) a_{d} / 2\right)^{d e g} \otimes\left(g(\rho, \psi)^{d-1}\right)^{d e g} \cong
$$

$\cong \mathcal{L}_{\psi\left((d-1) b_{d-1} t^{d-1}+(d-1) b_{0}\right)} \otimes \mathcal{L}_{\rho^{d}\left((-1)^{d}\left(s_{0} t\right)^{d-1}\right)} \otimes \rho^{d-1}\left(d(d-1) a_{d} / 2\right)^{\operatorname{deg}} \otimes\left(g(\rho, \psi)^{d-1}\right)^{\operatorname{deg} \cong}$

$\cong \mathcal{L}_{\psi\left((d-1) b_{d-1} t^{d-1}+(d-1) b_{0}\right)} \otimes \mathcal{L}_{\rho^{d(d-1)}\left(-s_{0} t\right)} \otimes \rho^{d}(-1)^{d e g} \otimes \rho^{d-1}\left(d(d-1) a_{d} / 2\right)^{\operatorname{deg}} \otimes\left(g(\rho, \psi)^{d-1}\right)^{d e g} \cong$

$\cong \mathcal{L}_{\psi\left((d-1) b_{d-1} t^{d-1}+(d-1) b_{0}\right)} \otimes \rho^{d}(-1)^{d e g} \otimes \rho^{d-1}\left(d(d-1) a_{d} / 2\right)^{d e g} \otimes\left(g(\rho, \psi)^{d-1}\right)^{d e g}$

since $\sum_{i=0}^{d-2}\left(\zeta^{j}\right)^{i}=0$ for $(d-1) \not j, d(d-1)$ is even and $\prod_{i=0}^{d-2} \zeta^{i}=(-1)^{d}$.

In particular, $[d-1]^{\star}\left(\operatorname{det} \mathcal{F}_{g}\right)$ and

$[d-1]^{\star} \mathcal{L}_{\psi\left((d-1) b_{d-1} t+(d-1) b_{0}\right)} \otimes \rho^{d}(-1)^{\operatorname{deg}} \otimes \rho^{d-1}\left(d(d-1) a_{d} / 2\right)^{\operatorname{deg}} \otimes\left(g(\rho, \psi)^{d-1}\right)^{\operatorname{deg}}$

are isomorphic characters of $D_{\infty}^{d-1}$, so there is some character $\chi: k^{\star} \rightarrow \overline{\mathbb{Q}}_{\ell}^{\star}$ with $\chi^{d-1}=1$ such that

$\operatorname{det} \mathcal{F}_{g} \cong \mathcal{L}_{\chi} \otimes \mathcal{L}_{\psi\left((d-1) b_{d-1} t+(d-1) b_{0}\right)} \otimes \rho^{d}(-1)^{\operatorname{deg}} \otimes \rho^{d-1}\left(d(d-1) a_{d} / 2\right)^{\operatorname{deg}} \otimes\left(g(\rho, \psi)^{d-1}\right)^{\operatorname{deg}}$

as representations of $D_{\infty}$. But then

$\left(\widehat{\operatorname{det} \mathcal{F}_{g}}\right) \otimes \mathcal{L}_{\chi} \otimes \mathcal{L}_{\psi\left((d-1) b_{d-1} t+(d-1) b_{0}\right)} \otimes \rho^{d}(-1)^{\operatorname{deg}} \otimes \rho^{d-1}\left(d(d-1) a_{d} / 2\right)^{d e g} \otimes\left(g(\rho, \psi)^{d-1}\right)^{\operatorname{deg}}$

is a rank 1 smooth sheaf on $\mathbb{G}_{m, k}$, tamely ramified at 0 and unramified at infinity, so it must be geometrically trivial, that is, $\chi$ is trivial (since everything else is unramified at 0). Moreover, since the Frobenius action is trivial at infinity it must be the trivial sheaf. Therefore

$\operatorname{det} \mathcal{F}_{g} \cong \mathcal{L}_{\psi\left((d-1) b_{d-1} t+(d-1) b_{0}\right)} \otimes \rho^{d}(-1)^{\operatorname{deg}} \otimes \rho^{d-1}\left(d(d-1) a_{d} / 2\right)^{\operatorname{deg}} \otimes\left(g(\rho, \psi)^{d-1}\right)^{\operatorname{deg}}$ as sheaves on $\mathbb{A}_{k}^{1}$.

Proposition 2.3. Suppose that $p>d$, the sheaf $\mathcal{F}_{g}$ does not have finite monodromy (e.g. $p>2 d-1$ ) and there do not exist $c, d \in k$ such that $g(x+c)+d$ is odd. Then we have an estimate

$$
\left|\sum_{x \in k_{r}} \psi\left(\operatorname{Tr}_{k_{r} / k}(f(x))\right)\right| \leq C_{d, r} q^{\frac{r+1}{2}}
$$

where

$$
C_{d, r}=\frac{1}{d-1} \sum_{i=0}^{d-1}|i-1|\left(\begin{array}{c}
d-2+r-i \\
r-i
\end{array}\right)\left(\begin{array}{c}
d-1 \\
i
\end{array}\right)
$$

unless $a_{d-1}=0$ and $r=d-1$, in which case there is an estimate

$$
\left|\sum_{x \in k_{r}} \psi\left(\operatorname{Tr}_{k_{r} / k}(f(x))\right)-(-1)^{d-1} q \cdot \rho^{d}(-1)\left(\psi\left(b_{0}\right) \rho\left(d(d-1) a_{d} / 2\right) g(\rho, \psi)\right)^{d-1}\right|<C_{d, r} q^{\frac{r+1}{2}} .
$$

Proof. By [4, Section 1], we have

$$
\begin{gathered}
\sum_{x \in k_{r}} \psi\left(\operatorname{Tr}_{k_{r} / k}(f(x))\right)=-\sum_{u \in k} \operatorname{Tr}\left(\operatorname{Frob}_{k, u} \mid\left[\mathcal{F}_{g}\right]_{u}^{r}\right)= \\
=\sum_{i=0}^{r}(-1)^{i-1}(i-1) \operatorname{Tr}\left(\operatorname{Frob}_{k}, \mathrm{H}_{c}^{1}\left(\mathbb{A}_{\bar{k}}^{1}, \operatorname{Sym}^{r-i} \mathcal{F}_{g} \otimes \wedge^{i} \mathcal{F}_{g}\right)\right)- \\
-\sum_{i=0}^{r}(-1)^{i-1}(i-1) \operatorname{Tr}\left(\operatorname{Frob}_{k}, \mathrm{H}_{c}^{2}\left(\mathbb{A}_{\bar{k}}, \operatorname{Sym}^{r-i} \mathcal{F}_{g} \otimes \wedge^{i} \mathcal{F}_{g}\right)\right)
\end{gathered}
$$

Let $G \subseteq \mathrm{GL}(V)$ be the geometric monodromy group of $\mathcal{F}_{g}$. Under the hypotheses of the proposition, the unit connected component of $G$ is $\operatorname{SL}(V)$, so $G$ is the inverse 
image of its image by the determinant. By lemma $2.2, G$ is $\operatorname{SL}(V)$ if $b_{d-1}=0$ (if and only if $a_{d-1}=0$ ) and $\mathrm{GL}_{p}(V)=\mu_{p} \cdot \mathrm{SL}(V)$ (since $p>d$, so $p$ does not divide $d-1)$ if $b_{d-1} \neq 0$.

For every $i$, the dimension of $\mathrm{H}_{c}^{2}\left(\mathbb{A}_{\bar{k}}, \operatorname{Sym}^{r-i} \mathcal{F}_{g} \otimes \wedge^{i} \mathcal{F}_{g}\right)$ is the dimension of the coinvariant (or the invariant) space of the action of $G$ on $\operatorname{Sym}^{r-i} V \otimes \wedge^{i} V$. By 15. Corollary 4.2], the action of $\mathrm{SL}(V) \subseteq G$ on $\operatorname{Sym}^{r-i} V \otimes \wedge^{i} V$ has no invariants unless $r=d-1$ and $i=r, r-1$, in which case the invariant space $W_{i}$ is onedimensional. If $a_{d-1} \neq 0$, a generator $\zeta_{p}$ of the quotient $G / \mathrm{SL}(V) \cong \mu_{p}$ acts on $W_{i}$ via multiplication by $\zeta_{p}^{d-1}$, which can not be trivial since $p>d$. So the action of $G$ has no invariants on $\operatorname{Sym}^{r-i} V \otimes \wedge^{i} V$ for any $i$ if $a_{d-1} \neq 0$.

In that case, since $\mathrm{H}_{c}^{1}\left(\mathbb{A}_{\bar{k}}^{1}, \operatorname{Sym}^{r-i} \mathcal{F}_{g} \otimes \wedge^{i} \mathcal{F}_{g}\right)$ is mixed of weights $\leq r+1$ we get

$$
\left|\sum_{x \in k_{r}} \psi\left(\operatorname{Tr}_{k_{r} / k}(f(x))\right)\right| \leq \sum_{i=0}^{r}|i-1| \operatorname{dim} \mathrm{H}_{c}^{1}\left(\mathbb{A}_{\bar{k}}^{1}, \operatorname{Sym}^{r-i} \mathcal{F}_{g} \otimes \wedge^{i} \mathcal{F}_{g}\right) \cdot q^{\frac{r+1}{2}} .
$$

Moreover, by the Ogg-Shafarevic formula we have

$$
\begin{gathered}
\operatorname{dim} \mathrm{H}_{c}^{1}\left(\mathbb{A}_{\bar{k}}^{\frac{1}{k}}, \operatorname{Sym}^{r-i} \mathcal{F}_{g} \otimes \wedge^{i} \mathcal{F}_{g}\right)=-\chi\left(\mathbb{A}_{\bar{k}}, \operatorname{Sym}^{r-i} \mathcal{F}_{g} \otimes \wedge^{i} \mathcal{F}_{g}\right)= \\
=\operatorname{Swan}_{\infty}\left(\operatorname{Sym}^{r-i} \mathcal{F}_{g} \otimes \wedge^{i} \mathcal{F}_{g}\right)-\operatorname{rank}\left(\operatorname{Sym}^{r-i} \mathcal{F}_{g} \otimes \wedge^{i} \mathcal{F}_{g}\right) \leq \\
\leq \frac{1}{d-1} \operatorname{rank}\left(\operatorname{Sym}^{r-i} \mathcal{F}_{g} \otimes \wedge^{i} \mathcal{F}_{g}\right)=\frac{1}{d-1}\left(\begin{array}{c}
d-2+r-i \\
r-i
\end{array}\right)\left(\begin{array}{c}
d-1 \\
i
\end{array}\right)
\end{gathered}
$$

since all slopes at infinity of $\mathcal{F}_{g}$ (and a fortiori of $\operatorname{Sym}^{r-i} \mathcal{F}_{g} \otimes \wedge^{i} \mathcal{F}_{g}$ ) are $\leq \frac{d}{d-1}$.

Suppose now that $a_{d-1}=0$ and $r=d-1$. As in [15, Corollary 4.2], we have

$$
\begin{gathered}
\sum_{i=0}^{r}(-1)^{i-1}(i-1) \operatorname{Tr}\left(\operatorname{Frob}_{k}, \mathrm{H}_{c}^{2}\left(\mathbb{A}_{\bar{k}}^{1}, \operatorname{Sym}^{r-i} \mathcal{F}_{g} \otimes \wedge^{i} \mathcal{F}_{g}\right)\right)= \\
=(-1)^{r}(r-2) \operatorname{Tr}\left(\operatorname{Frob}_{k}, \mathrm{H}_{c}^{2}\left(\mathbb{A}_{\bar{k}}^{1}, \operatorname{Sym}^{1} \mathcal{F}_{g} \otimes \wedge^{r-1} \mathcal{F}_{g}\right)\right)+ \\
+(-1)^{r-1}(r-1) \operatorname{Tr}\left(\operatorname{Frob}_{k}, \mathrm{H}_{c}^{2}\left(\mathbb{A}_{\bar{k}}, \wedge^{r} \mathcal{F}_{g}\right)\right)= \\
=(-1)^{r-1} \operatorname{Tr}\left(\operatorname{Frob}_{k}, \mathrm{H}_{c}^{2}\left(\mathbb{A}_{\bar{k}}^{1}, \operatorname{det} \mathcal{F}_{g}\right)\right)= \\
=(-1)^{d} q \cdot \psi\left((d-1) b_{0}\right) \rho^{d}(-1) \rho^{d-1}\left(d(d-1) a_{d} / 2\right) g(\rho, \psi)^{d-1}= \\
=(-1)^{d} q \cdot \rho^{d}(-1)\left(\psi\left(b_{0}\right) \rho\left(d(d-1) a_{d} / 2\right) g(\rho, \psi)\right)^{d-1}
\end{gathered}
$$

by lemma 2.2. We conclude as above using the fact that, for the two values of $i$ for which $\mathrm{H}_{c}^{2}\left(\mathbb{A}_{\bar{k}}^{1}, \operatorname{Sym}^{r-i} \mathcal{F}_{g} \otimes \wedge^{i} \mathcal{F}_{g}\right)$ is one-dimensional, the sheaf $\operatorname{Sym}^{r-i} \mathcal{F}_{g} \otimes \wedge^{i} \mathcal{F}_{g}$ has at least one slope equal to 0 at infinity, so

$$
\begin{gathered}
\operatorname{dim} \mathrm{H}_{c}^{1}\left(\mathbb{A}_{\bar{k}}^{\frac{1}{k}}, \operatorname{Sym}^{r-i} \mathcal{F}_{g} \otimes \wedge^{i} \mathcal{F}_{g}\right)=1-\chi\left(\mathbb{A}_{\bar{k}}^{\frac{1}{k}}, \operatorname{Sym}^{r-i} \mathcal{F}_{g} \otimes \wedge^{i} \mathcal{F}_{g}\right)= \\
=1+\operatorname{Swan}_{\infty}\left(\operatorname{Sym}^{r-i} \mathcal{F}_{g} \otimes \wedge^{i} \mathcal{F}_{g}\right)-\operatorname{rank}\left(\operatorname{Sym}^{r-i} \mathcal{F}_{g} \otimes \wedge^{i} \mathcal{F}_{g}\right) \leq \\
\leq 1+\frac{d}{d-1}\left(\operatorname{rank}\left(\operatorname{Sym}^{r-i} \mathcal{F}_{g} \otimes \wedge^{i} \mathcal{F}_{g}\right)-1\right)-\operatorname{rank}\left(\operatorname{Sym}^{r-i} \mathcal{F}_{g} \otimes \wedge^{i} \mathcal{F}_{g}\right)< \\
<\frac{1}{d-1} \operatorname{rank}\left(\operatorname{Sym}^{r-i} \mathcal{F}_{g} \otimes \wedge^{i} \mathcal{F}_{g}\right)=\frac{1}{d-1}\left(\begin{array}{c}
d-2+r-i \\
r-i
\end{array}\right)\left(\begin{array}{c}
d-1 \\
i
\end{array}\right) .
\end{gathered}
$$


Proposition 2.4. Suppose that $p>d$, the sheaf $\mathcal{F}_{g}$ does not have finite monodromy (e.g. $p>2 d-1$ ) and there exist $\alpha, \beta \in k$ such that $g(x+\alpha)+\beta$ is odd (so $d$ is odd). Then we have an estimate

$$
\left|\sum_{x \in k_{r}} \psi\left(\operatorname{Tr}_{k_{r} / k}(f(x))\right)\right| \leq C_{d, r} q^{\frac{r+1}{2}}
$$

where

$$
C_{d, r}=\frac{1}{d-1} \sum_{i=0}^{d-1}|i-1|\left(\begin{array}{c}
d-2+r-i \\
r-i
\end{array}\right)\left(\begin{array}{c}
d-1 \\
i
\end{array}\right)
$$

unless $a_{d-1}=0$ and $r \leq d-1$ is even, in which case there is an estimate

$$
\left|\sum_{x \in k_{r}} \psi\left(\operatorname{Tr}_{k_{r} / k}(f(x))\right)-(-1)^{r} \psi(-\beta)^{r} q^{\frac{r}{2}+1}\right|<C_{d, r} q^{\frac{r+1}{2}} .
$$

Proof. The proof is similar to the previous one. In this case, the unit connected component of $G$ is $\operatorname{Sp}(V)$, so by lemma $2.2 G$ is $\operatorname{Sp}(V)$ if $b_{d-1}=0$ (if and only if $a_{d-1}=0$ ) and $\mu_{p} \cdot \operatorname{SL}(V)$ (since $p>d$, so $p$ does not divide $d-1$ ) if $b_{d-1} \neq 0$.

By [10, lemma on p.62], the action of $\operatorname{Sp}(V) \subseteq G$ on $\operatorname{Sym}^{r-i} V \otimes \wedge^{i} V$ has no invariants unless $r \leq d-1$ is even and $i=r, r-1$, in which case the invariant space $W_{i}$ is one-dimensional. If $a_{d-1} \neq 0$, a generator $\zeta_{p}$ of the quotient $G / \operatorname{Sp}(V) \cong \mu_{p}$ acts on $W_{i}$ via multiplication by $\zeta_{p}^{d-1}$, which can not be trivial since $p>d$. So the action of $G$ has no invariants on $\operatorname{Sym}^{r-i} V \otimes \wedge^{i} V$ for any $i$ if $a_{d-1} \neq 0$. We conclude this case as in the previous proposition.

Suppose now that $a_{d-1}=0, r \leq d-1$ is even and $i=r$ or $r-1$. Since the coefficient of $x^{d-1}$ in $g(x)$ is 0 , the coefficient in $g(x+\alpha)+\beta$ is $d a_{d} \alpha$, so it can only be an odd polynomial if $\alpha=0$. That is, $g(x)+\beta$ is odd, or equivalently, $g(-x)=-2 \beta-g(x)$. Then the sheaf $\psi(\beta)^{\operatorname{deg}} \otimes \mathcal{F}_{g}(1 / 2)$ is self-dual: since the dual of $\mathcal{L}_{\psi(g)}$ is $\mathcal{L}_{\psi(-g)}(1)$, using that $D \circ F T_{\psi}=[-1]^{\star} F T_{\psi} \circ D(1)$ [13, Corollaire 2.1.5] we get that the dual of $\mathcal{F}_{g}=\mathcal{H}^{-1}\left(F T_{\psi}\left(\mathcal{L}_{\psi(g)}[1]\right)\right)$ is

$[-1]^{\star} \mathcal{H}^{-1}\left(F T_{\psi} \mathcal{L}_{\psi(-g)}(1)\right)=[-1]^{\star} \mathcal{F}_{-g}(1)=[-1]^{\star} \mathcal{F}_{2 \beta+g(-x)}(1)=\psi(2 \beta)^{\operatorname{deg}} \otimes \mathcal{F}_{g}(1)$

so $\psi(\beta)^{\text {deg }} \otimes \mathcal{F}_{g}(1 / 2)$ is self-dual (symplectically, since it is so geometrically by [7. Theorem 19]). In particular, the one-dimensional $\operatorname{Sp}(V)$-invariant subspace of $\left(\operatorname{Sym}^{r-i} \mathcal{F}_{g} \otimes \wedge^{i} \mathcal{F}_{g}\right) \otimes \psi(\beta)^{r \cdot \operatorname{deg}}(r / 2)$ is also invariant under all Frobenii. So $W_{i}$ is in fact the geometrically constant sheaf $\psi(-\beta)^{r \cdot d e g}(-r / 2)$. In particular

$$
\begin{gathered}
\sum_{i=0}^{r}(-1)^{i-1}(i-1) \operatorname{Tr}\left(\operatorname{Frob}_{k}, \mathrm{H}_{c}^{2}\left(\mathbb{A}_{\bar{k}}^{1}, \operatorname{Sym}^{r-i} \mathcal{F}_{g} \otimes \wedge^{i} \mathcal{F}_{g}\right)\right)= \\
=(-1)^{r}(r-2) \operatorname{Tr}\left(\operatorname{Frob}_{k}, \mathrm{H}_{c}^{2}\left(\mathbb{A}_{\bar{k}}^{1}, \operatorname{Sym}^{1} \mathcal{F}_{g} \otimes \wedge^{r-1} \mathcal{F}_{g}\right)\right)+ \\
+(-1)^{r-1}(r-1) \operatorname{Tr}\left(\operatorname{Frob}_{k}, \mathrm{H}_{c}^{2}\left(\mathbb{A}_{\bar{k}}^{1}, \wedge^{r} \mathcal{F}_{g}\right)\right)= \\
=(-1)^{r-1} \operatorname{Tr}\left(\operatorname{Frob}_{k}, \mathrm{H}_{c}^{2}\left(\mathbb{A}_{\bar{k}}, \psi(-\beta)^{r \cdot d e g}(-r / 2)\right)\right)=(-1)^{r-1} \psi(-\beta)^{r} q^{\frac{r}{2}+1} .
\end{gathered}
$$

We conclude as in the previous proposition. 


\section{MULTIPLICATIVE CHARACTER SUMS FOR TRANSLATION INVARIANT POLYNOMIALS}

Let $f \in k[x]$ be translation invariant, and $g \in k[x]$ of degree $d$ such that $f(x)=$ $g\left(x^{q}-x\right)$. Let $\chi: k^{\star} \rightarrow \overline{\mathbb{Q}}_{\ell}^{\star}$ a non-trivial multiplicative character of order $m$, extended by zero to all of $k$. Since $f$ has degree $q d$, Weil's bound gives in this case

$$
\left|\sum_{x \in k_{r}} \chi\left(\mathrm{N}_{k_{r} / k}(f(x))\right)\right| \leq(q d-1) q^{\frac{r}{2}} .
$$

On the other hand we have, for every $r \geq 1$,

$$
\begin{gathered}
\sum_{x \in k_{r}} \chi\left(\mathrm{N}_{k_{r} / k}(f(x))\right)=\sum_{x \in k_{r}} \chi\left(\mathrm{N}_{k_{r} / k}\left(g\left(x^{q}-x\right)\right)\right)= \\
=\sum_{t \in k_{r}} \#\left\{x \in k_{r} \mid x^{q}-x=t\right\} \chi\left(\mathrm{N}_{k_{r} / k}(g(t))\right)= \\
=\sum_{t \in k_{r}} \sum_{u \in k} \psi\left(u \operatorname{Tr}_{k_{r} / k}(t)\right) \chi\left(\mathrm{N}_{k_{r} / k}(g(t))\right)=\sum_{u \in k} \sum_{t \in k_{r}} \psi\left(u \operatorname{Tr}_{k_{r} / k}(t)\right) \chi\left(\mathrm{N}_{k_{r} / k}(g(t))\right) .
\end{gathered}
$$

Consider the $\overline{\mathbb{Q}}_{\ell}$-sheaf $\mathcal{L}_{\chi(g)}:=g^{\star} \mathcal{L}_{\chi}$ on $\mathbb{A}_{k}^{1}$, where $\mathcal{L}_{\chi}$ is the Kummer sheaf on $\mathbb{G}_{m, k}$ associated to $\chi[1,1.7]$, extended by zero to $\mathbb{A}_{k}^{1}$. Suppose that $g$ is square-free and its degree $d$ is prime to $p$. Then $\mathcal{L}_{\chi(g)}$ is an irreducible middle extension sheaf, smooth on the complement of the subscheme $Z \subseteq \mathbb{A}_{k}^{1}$ defined by $g=0$. Since there is at least one point where it is not smooth, it is not isomorphic to an Artin-Schreier sheaf and therefore the Fourier transform of $\mathcal{L}_{\chi(g)}[1]$ is a single irreducible middle extension sheaf $\mathcal{F}_{g}$ placed in degree -1 [8, 8.2]. We have

$$
\begin{gathered}
\sum_{x \in k_{r}} \chi\left(\mathrm{N}_{k_{r} / k}(f(x))\right)=\sum_{x \in k_{r}} \chi\left(\mathrm{N}_{k_{r} / k}\left(g\left(x^{q}-x\right)\right)\right)=\sum_{u \in k} \sum_{t \in k_{r}} \psi\left(u \operatorname{Tr}_{k_{r} / k}(t)\right) \chi\left(\mathrm{N}_{k_{r} / k}(g(t))\right)= \\
=-\sum_{u \in k} \operatorname{Tr}\left(\operatorname{Frob}_{k, u}^{r} \mid\left(\mathcal{F}_{g}\right)_{u}\right)=-\sum_{u \in k} \operatorname{Tr}\left(\operatorname{Frob}_{k, u} \mid\left[\mathcal{F}_{g}\right]_{u}^{r}\right)
\end{gathered}
$$

where $\left[\mathcal{F}_{g}\right]^{r}$ is the $r$-th Adams power of $\mathcal{F}_{g}$.

Proposition 3.1. The sheaf $\mathcal{F}_{g}$ has generic rank $d$, it is smooth on $\mathbb{G}_{m, k}$ and tamely ramified at 0 . Its rank at 0 is $d-1$. If all roots of $g$ are in $k$, the action of the decomposition group $D_{\infty}$ on the generic stalk of $\mathcal{F}_{g}$ splits as a direct sum $\bigoplus_{a} \chi\left(g^{\prime}(a)\right)^{\text {deg }} \otimes g(\chi, \psi)^{\text {deg }} \otimes \mathcal{L}_{\bar{\chi}} \otimes \mathcal{L}_{\psi_{a}}$ where the sum is taken over the roots of $f, \mathcal{L}_{\psi_{a}}$ is the Artin-Schreier sheaf corresponding to the character $t \mapsto \psi($ at $)$ and $g(\chi, \psi)=-\sum_{t} \chi(t) \psi(t)$ if the Gauss sum.

Proof. The generic rank of $\mathcal{F}_{g}$ is the dimension of $\mathrm{H}_{c}^{1}\left(\mathbb{A}_{\frac{1}{k}}, \mathcal{L}_{\chi(g)} \otimes \mathcal{L}_{\psi_{z}}\right)$ for generic $z$. Since $\mathcal{L}_{\chi(g)}$ is tamely ramified everywhere and has rank one, for any $z \neq 0$ $\mathcal{L}_{\chi(g)} \otimes \mathcal{L}_{\psi_{z}}$ is tamely ramified at every point of $\mathbb{A}_{\bar{k}}$ and totally wild at infinity with Swan conductor 1. In particular its $\mathrm{H}_{c}^{i}$ vanish for $i \neq 1$. By the Ogg-Shafarevic formula, its Euler characteristic is then $1-d-1=-d$, since there are $d$ points in $\mathbb{A}_{\bar{k}} \frac{1}{k}$ where the stalk is zero. Therefore $\operatorname{dim} \mathrm{H}_{c}^{1}\left(\mathbb{A}_{\bar{k}}^{1}, \mathcal{L}_{\chi(g)} \otimes \mathcal{L}_{\psi_{z}}\right)=d$ for every $z \neq 0$. Similarly, it is $d-1$ for $z=0$. Since $\mathcal{F}_{g}$ is a middle extension, it is smooth exactly on the open set where the rank is maximal, so it is smooth on $\mathbb{G}_{m, k}$. It is tamely ramified at zero, since $\mathcal{L}_{\chi(g)}$ is tamely ramified at infinity [14, Théorème 2.4.3].

Suppose now that all roots of $g$ are in $k$, and let $a$ be one such root. In an étale neighborhood of $a$, the sheaf $\mathcal{L}_{\chi(g)}$ is isomorphic to $\mathcal{L}_{\chi\left(g^{\prime}(a)(x-a)\right)}=\chi\left(g^{\prime}(a)\right)^{\text {deg }} \otimes$ 
$\mathcal{L}_{\chi(x-a)}$, since $g(x)=g^{\prime}(a)(x-a) \frac{g(x)}{g^{\prime}(a)(x-a)}$ and $\frac{g(x)}{g^{\prime}(a)(x-a)}$ is an $m$-th power in the henselization of $\mathbb{A}_{k}^{1}$ at $a$ (since its image in the residue field is 1). Applying Laumon's local Fourier transform [14, Proposition 2.5.3.1] and using that Fourier transform commutes with tensoring by unramified sheaves, we deduce that the $D_{\infty}$-representation $\mathcal{F}_{g}$ contains $\left(L F T_{\psi}^{(0, \infty)} \chi\left(g^{\prime}(a)\right)^{\text {deg }} \otimes \mathcal{L}_{\chi}\right) \otimes \mathcal{L}_{\psi_{a}}=\chi\left(g^{\prime}(a)\right)^{\text {deg }} \otimes$ $g(\chi, \psi)^{\text {deg }} \otimes \mathcal{L}_{\bar{\chi}} \otimes \mathcal{L}_{\psi_{a}}$ as a direct summand. Since $g$ has $d$ distinct roots we obtain $d$ different terms this way, which is the rank of $\mathcal{F}_{g}$, so its monodromy at $\infty$ is the direct sum of these terms.

Define by induction the sequence of polynomials $g_{n}[x] \in k[x]$ for $n \geq 1$ by: $g_{1}(x)=g(x)$, and for $n \geq 1 g_{n+1}(x)$ is the resultant in $t$ of $g_{n}(t)$ and $g(x-t)$.

Corollary 3.2. Suppose that either $m$ does not divide $r$ or $g_{r}(0) \neq 0$. Then we have an estimate

$$
\left|\sum_{x \in k_{r}} \chi\left(\mathrm{N}_{k_{r} / k}(f(x))\right)\right| \leq C_{d, r} q^{\frac{r+1}{2}}
$$

where

$$
C_{d, r}=\sum_{i=0}^{r}|i-1|\left(\left(\begin{array}{c}
d-1+r-i \\
r-i
\end{array}\right)\left(\begin{array}{l}
d \\
i
\end{array}\right)-\left(\begin{array}{c}
d-2+r-i \\
r-i
\end{array}\right)\left(\begin{array}{c}
d-1 \\
i
\end{array}\right)\right) .
$$

Proof. By the previous proposition, the action of the inertia group $I_{\infty}$ on $\mathcal{F}_{g}^{\otimes r}$ splits as a direct sum over the $r$-uples of roots of $f$

$$
\bigoplus \mathcal{L}_{\bar{\chi}}^{\otimes r} \otimes \mathcal{L}_{\left.\psi_{a_{1}}, \ldots, a_{r}\right)} \otimes \cdots \otimes \mathcal{L}_{\psi_{a_{r}}}=\bigoplus_{\left(a_{1}, \ldots, a_{r}\right)} \mathcal{L}_{\bar{\chi}}^{\otimes r} \otimes \mathcal{L}_{\psi_{a_{1}+\cdots+a_{r}}}
$$

For each $\left(a_{1}, \ldots, a_{r}\right)$, the character $\mathcal{L}_{\bar{\chi}}^{\otimes r} \otimes \mathcal{L}_{\psi_{a_{1}+\cdots+a_{r}}}$ is trivial if and only if both $\mathcal{L}_{\bar{\chi}}^{\otimes r}$ and $\mathcal{L}_{\psi_{a_{1}+\cdots+a_{r}}}$ are trivial, that is, if and only if $m$ divides $r$ and $a_{1}+\cdots+$ $a_{r}=0$. Under the hypotheses of the corollary, at least one of these conditions does not hold (since the sums $a_{1}+\cdots+a_{r}$ are the roots of $g_{r}$ ). So $\mathcal{F}_{g}^{\otimes r}$ has no invariants under the action of $I_{\infty}$ and, a fortiori, under the action of the larger group $\pi_{1}\left(\mathbb{G}_{m, \bar{k}}, \bar{\eta}\right)$. Since $\operatorname{Sym}^{r-i} \mathcal{F}_{g} \otimes \wedge^{i} \mathcal{F}_{g}$ is a subsheaf of $\mathcal{F}_{g}^{\otimes r}$ for every $i$, we conclude that $\mathrm{H}_{c}^{2}\left(\mathbb{A}_{\bar{k}}, \operatorname{Sym}^{r-i} \mathcal{F}_{g} \otimes \wedge^{i} \mathcal{F}_{g}\right)=0$ for every $i=0, \ldots, r$. Therefore

$$
\begin{gathered}
\sum_{x \in k_{r}} \chi\left(\mathrm{N}_{k_{r} / k}(f(x))\right)=-\sum_{u \in k} \operatorname{Tr}\left(\operatorname{Frob}_{k, u} \mid\left[\mathcal{F}_{g}\right]_{u}^{r}\right)= \\
=\sum_{i=0}^{r}(-1)^{i-1}(i-1) \operatorname{Tr}\left(\operatorname{Frob}_{k}, \mathrm{H}_{c}^{1}\left(\mathbb{A}_{k}, \operatorname{Sym}^{r-i} \mathcal{F}_{g} \otimes \wedge^{i} \mathcal{F}_{g}\right)\right) .
\end{gathered}
$$

Since $\mathrm{H}_{c}^{1}\left(\mathbb{A}_{\frac{1}{k}}, \operatorname{Sym}^{r-i} \mathcal{F}_{g} \otimes \wedge^{i} \mathcal{F}_{g}\right)$ is mixed of weights $\leq r+1$, we get

$$
\left|\sum_{x \in k_{r}} \chi\left(\mathrm{N}_{k_{r} / k}(f(x))\right)\right| \leq \sum_{i=0}^{r}|i-1| \operatorname{dim} \mathrm{H}_{c}^{1}\left(\mathbb{A}_{\bar{k}}, \operatorname{Sym}^{r-i} \mathcal{F}_{g} \otimes \wedge^{i} \mathcal{F}_{g}\right) \cdot q^{\frac{r+1}{2}} \text {. }
$$

And by the Ogg-Shafarevic formula, we have

$$
\begin{gathered}
\operatorname{dim} \mathrm{H}_{c}^{1}\left(\mathbb{A}_{\frac{1}{k}}, \operatorname{Sym}^{r-i} \mathcal{F}_{g} \otimes \wedge^{i} \mathcal{F}_{g}\right)=-\chi\left(\mathbb{A}_{k}^{\frac{1}{k}}, \operatorname{Sym}^{r-i} \mathcal{F}_{g} \otimes \wedge^{i} \mathcal{F}_{g}\right)= \\
=\operatorname{Swan}_{\infty}\left(\operatorname{Sym}^{r-i} \mathcal{F}_{g} \otimes \wedge^{i} \mathcal{F}_{g}\right)-\operatorname{rank}_{0}\left(\operatorname{Sym}^{r-i} \mathcal{F}_{g} \otimes \wedge^{i} \mathcal{F}_{g}\right) \leq \\
\leq\left(\begin{array}{c}
d-1+r-i \\
r-i
\end{array}\right)\left(\begin{array}{c}
d \\
i
\end{array}\right)-\left(\begin{array}{c}
d-2+r-i \\
r-i
\end{array}\right)\left(\begin{array}{c}
d-1 \\
i
\end{array}\right)
\end{gathered}
$$


by the previous proposition, since $\mathcal{F}_{g}$ is smooth on $\mathbb{G}_{m, k}$, tamely ramified at 0 and all its slopes at infinity (and thus all slopes of of $\operatorname{Sym}^{r-i} \mathcal{F}_{g} \otimes \wedge^{i} \mathcal{F}_{g}$ ) are $\leq 1$.

Corollary 3.3. If all roots of $g(x)=\sum_{i=0}^{d} a_{i} x^{i}$ are in $k$, the determinant of $\mathcal{F}_{g}$ is $\chi\left((-1)^{d(d-1) / 2} a_{d}^{-(d-2)} \operatorname{disc}(g)\right)^{\operatorname{deg}} \otimes\left(g(\chi, \psi)^{d}\right)^{\operatorname{deg}} \otimes \mathcal{L}_{\bar{\chi}^{d}} \otimes \mathcal{L}_{\psi_{-a_{d-1} / a_{d}}}$.

Proof. By proposition 3.1. the action of $D_{\infty}$ on the determinant of $\mathcal{F}_{g}$ is given by $\mathcal{G}:=\bigotimes_{a} \chi\left(g^{\prime}(a)\right)^{\operatorname{deg}} \otimes g(\chi, \psi)^{\operatorname{deg}} \otimes \mathcal{L}_{\bar{\chi}} \otimes \mathcal{L}_{\psi_{a}}=\chi\left(\prod_{a} g^{\prime}(a)\right)^{\operatorname{deg}} \otimes\left(g(\chi, \psi)^{d}\right)^{\operatorname{deg}} \otimes \mathcal{L}_{\bar{\chi}^{d}} \otimes \mathcal{L}_{\psi_{\Sigma a}}$

where the product is taken over the roots of $g$. Now $\sum a=-a_{d-1} / a_{d}$, and

$$
\begin{gathered}
\prod_{a} g^{\prime}(a)=\prod_{a} a_{d} \prod_{g(b)=0, b \neq a}(b-a)= \\
=a_{d}^{d} \prod_{g(a)=g(b)=0, a \neq b}(a-b)=(-1)^{d(d-1) / 2} a_{d}^{-(d-2)} \operatorname{disc}(g) .
\end{gathered}
$$

Therefore $\operatorname{det}\left(\mathcal{F}_{g}\right) \otimes \hat{\mathcal{G}}$ is smooth on $\mathbb{G}_{m, k}$, tamely ramified at zero and unramified at infinity, so it is geometrically constant. Looking at the Frobenius action at 0 , it must be the constant sheaf $\overline{\mathbb{Q}}_{\ell}$. We conclude that $\operatorname{det}\left(\mathcal{F}_{g}\right) \cong \mathcal{G}$.

Proposition 3.4. Let $h(x)=g\left(x-\frac{a_{d-1}}{d a_{d}}\right)$. Suppose that $p>2 d+1$ and $h$ is not odd (for $d$ odd) or even (for $d$ even). Then the geometric monodromy group $G$ of $\mathcal{F}_{g}$ is $\mathrm{GL}_{s p}(V)$ if $a_{d-1} \neq 0$ and $\mathrm{GL}_{s}(V)$ if $a_{d-1}=0$, where $V$ is the geometric generic stalk of $\mathcal{F}_{g}$ and $s$ is the order of $\chi^{d}$.

Proof. Since $\mathcal{L}_{\chi(g)}$ is the translate of $\mathcal{L}_{\chi(h)}$ by $a:=\frac{a_{d-1}}{d a_{d}}$, we have $\mathcal{F}_{g}=\mathcal{F}_{h} \otimes \mathcal{L}_{\psi_{a}}$. If $G$ (respectively $G^{\prime}$ ) is the geometric monodromy group of $\mathcal{F}_{g}$ (resp. $\mathcal{F}_{h}$ ), we have then $G \subseteq \mu_{p} \cdot G^{\prime}$ and $G^{\prime} \subseteq \mu_{p} \cdot G$. In particular, the unit connected components $G_{0}$ and $G_{0}^{\prime}$ are the same. Since $\mathcal{F}_{g}$ is pure, $G_{0}$ is a semisimple group 2, Corollaire 1.3.9], so by [9, Theorem 7.6.3.1], $\mathcal{F}_{g}$ is Lie-irreducible and $G_{0}$ is one of $\operatorname{SL}(V)$, $\mathrm{Sp}(V)$ (only possible if $\chi^{d}=\mathbf{1}$ ) or $\mathrm{SO}(V)$ (only possible if $\chi^{d}$ has order 2). We will see that, under the given hypotheses, the last two options are not possible.

By corollary 3.3 the determinant of $\mathcal{F}_{h}$ is geometrically isomorphic to $\mathcal{L}_{\bar{\chi}^{d}}$. By [7. Proposition 6], the factor group $G^{\prime} / G_{0}^{\prime}$ is cyclic of finite prime to $p$ order. In particular, there exists some prime to $p$ integer $e$ such that the geometric monodromy group of the pull-back $[e]^{\star} \mathcal{F}_{h}$ is in $G_{0}^{\prime}$, where $[e]: \mathbb{G}_{m, k} \rightarrow \mathbb{G}_{m, k}$ is the $e$-th power map. If $G_{0}^{\prime}=\mathrm{Sp}(V)$ or $\mathrm{SO}(V),[e]^{\star} \mathcal{F}_{h}$ would then be geometrically self-dual. By proposition 3.1, its restriction to the inertia group $I_{\infty}$ is the direct sum of $[e]^{\star} \mathcal{L}_{\psi_{b}} \otimes \mathcal{L}_{\bar{\chi}^{e}}$ taken over the roots $b$ of $h$. Its dual is then the direct sum on $[e]^{\star} \mathcal{L}_{\psi_{-b}} \otimes \mathcal{L}_{\chi^{e}}$. Given that the dual of $[e]^{\star} \mathcal{L}_{\psi_{b}}$ is $[e]^{\star} \mathcal{L}_{\psi_{-b}}$, in order for this to be self-dual as a representation of $I_{\infty}$ a necessary condition is that the set of roots of $h$ is symmetric with respect to 0 , that is, that $h$ is either even or odd (since it is a priori square-free).

So, if $h$ is neither even nor odd, $G_{0}$ is $\operatorname{SL}(V)$. Then $G$ is $\operatorname{SL}_{n}(V)$, where $n$ is the geometric order of the determinant of $\mathcal{F}_{g}$. By corollary 3.3. this order is $s p$ if $a_{d-1} \neq 0$ and $s$ if $a_{d-1}=0$. 
Corollary 3.5. Let $h(x)=g\left(x-\frac{a_{d-1}}{d a_{d}}\right)$. Suppose that $p>2 d+1$ and $h$ is not odd (for $d$ odd) or even (for d even). Then we have an estimate

$$
\left|\sum_{x \in k_{r}} \chi\left(\mathrm{N}_{k_{r} / k}(f(x))\right)\right| \leq C_{d, r} q^{\frac{r+1}{2}}
$$

where

$$
C_{d, r}=\sum_{i=0}^{r}|i-1|\left(\left(\begin{array}{c}
d-1+r-i \\
r-i
\end{array}\right)\left(\begin{array}{l}
d \\
i
\end{array}\right)-\left(\begin{array}{c}
d-2+r-i \\
r-i
\end{array}\right)\left(\begin{array}{c}
d-1 \\
i
\end{array}\right)\right)
$$

unless $r=d, \chi^{d}$ is trivial and $a_{d-1}=0$, in which case there exists an $\ell$-adic unit $\beta \in \overline{\mathbb{Q}}_{\ell}$ with $|\beta|=q^{\frac{d}{2}}$ such that

$$
\left|\sum_{x \in k_{r}} \chi\left(\mathrm{N}_{k_{r} / k}(f(x))\right)-(-1)^{d} q \beta\right| \leq C_{d, r} q^{\frac{r+1}{2}} .
$$

If $k$ contains all roots of $g$, then $\beta=\chi\left((-1)^{d(d-1) / 2} a_{d}^{-(d-2)} \operatorname{disc}(g)\right) g(\chi, \psi)^{d}$.

Proof. By the previous proposition, the monodromy group $G$ of $\mathcal{F}_{g}$ is $\mathrm{GL}_{s p}(V)$ if $a_{d-1} \neq 0$ and $\mathrm{GL}_{s}(V)$ if $a_{d-1}=0$. We proceed as in the proof of proposition 2.3 . $G_{0}$ has no invariants on $\operatorname{Sym}^{r-i} V \otimes \wedge^{i} V$ unless $r=d$ and $i=r, r-1$, in which case the invariant space is one-dimensional and $G$ acts on it via multiplication by the determinant. So the action of $G$ does not have invariants unless $a_{d-1}=0$ and $\chi^{d}$ is trivial (i.e. $m \mid d$ ) by corollary 3.3 . In that case we obtain the estimate as in 2.3 . using the value for $C_{d, r}$ computed in corollary 3.2 .

In the exceptional case, we have again

$$
\begin{gathered}
\sum_{i=0}^{r}(-1)^{i-1}(i-1) \operatorname{Tr}\left(\operatorname{Frob}_{k}, \mathrm{H}_{c}^{2}\left(\mathbb{A}_{\bar{k}}^{1}, \operatorname{Sym}^{r-i} \mathcal{F}_{g} \otimes \wedge^{i} \mathcal{F}_{g}\right)\right)= \\
=(-1)^{r-1} \operatorname{Tr}\left(\operatorname{Frob}_{k}, \mathrm{H}_{c}^{2}\left(\mathbb{A}_{\bar{k}}^{1}, \operatorname{det} \mathcal{F}_{g}\right)\right) .
\end{gathered}
$$

Now $\operatorname{det} \mathcal{F}_{g}$ is geometrically constant of weight $d$, so there exists an $\ell$-adic unit $\beta$ with $|\beta|=1$ such that $\operatorname{det} \mathcal{F}_{g}=\left(\beta q^{\frac{d}{2}}\right)^{\operatorname{deg}}$. Then $\operatorname{Tr}\left(\operatorname{Frob}_{k}, \mathrm{H}_{c}^{2}\left(\mathbb{A}_{\bar{k}}, \operatorname{det} \mathcal{F}_{g}\right)\right)=$ $\beta q^{\frac{d}{2}+1}$. If $k$ contains all roots of $g$, the value of $\beta$ is given in corollary 3.3 . We conclude as in proposition 2.3 using that, for the two values of $i$ for which $\mathrm{H}_{c}^{2}\left(\mathbb{A}_{\bar{k}}^{1}, \operatorname{Sym}^{r-i} \mathcal{F}_{g} \otimes \wedge^{i} \mathcal{F}_{g}\right)$ is one-dimensional, the sheaf $\operatorname{Sym}^{r-i} \mathcal{F}_{g} \otimes \wedge^{i} \mathcal{F}_{g}$ has at least one slope equal to 0 at infinity, so

$$
\begin{gathered}
\operatorname{dim} \mathrm{H}_{c}^{1}\left(\mathbb{A}_{\bar{k}}^{1}, \operatorname{Sym}^{r-i} \mathcal{F}_{g} \otimes \wedge^{i} \mathcal{F}_{g}\right)=1-\chi\left(\mathbb{A}_{\bar{k}}^{1}, \operatorname{Sym}^{r-i} \mathcal{F}_{g} \otimes \wedge^{i} \mathcal{F}_{g}\right)= \\
=1+\operatorname{Swan}_{\infty}\left(\operatorname{Sym}^{r-i} \mathcal{F}_{g} \otimes \wedge^{i} \mathcal{F}_{g}\right)-\operatorname{rank}_{0}\left(\operatorname{Sym}^{r-i} \mathcal{F}_{g} \otimes \wedge^{i} \mathcal{F}_{g}\right) \leq \\
\leq \operatorname{gen} \cdot \operatorname{rank}\left(\operatorname{Sym}^{r-i} \mathcal{F}_{g} \otimes \wedge^{i} \mathcal{F}_{g}\right)-\operatorname{rank}_{0}\left(\operatorname{Sym}^{r-i} \mathcal{F}_{g} \otimes \wedge^{i} \mathcal{F}_{g}\right)= \\
=\left(\begin{array}{c}
d-1+r-i \\
r-i
\end{array}\right)\left(\begin{array}{c}
d \\
i
\end{array}\right)-\left(\begin{array}{c}
d-2+r-i \\
r-i
\end{array}\right)\left(\begin{array}{c}
d-1 \\
i
\end{array}\right) .
\end{gathered}
$$




\section{AdDITIVE CHARACTER SUMS FOR HOMOTHETY INVARIANT POLYNOMIALS}

Let $f \in k_{r}[x]$ be a polynomial and $e \mid q-1$ an integer. Let $\Gamma_{e} \subseteq k^{\star}$ be the unique subgroup of $k^{\star}$ of index $e$. We say that $f$ is $\Gamma_{e}$-homothety invariant if $f(\lambda x)=f(x)$ for every $\lambda \in \Gamma_{e}$. Equivalently, if $f\left(\lambda^{e} x\right)=f(x)$ for every $\lambda \in k^{\star}$. An argument similar to that in lemma 2.1 shows

Lemma 4.1. Let $f \in k_{r}[x]$ and $e \mid q-1$. The following conditions are equivalent:

(a) $f$ is $\Gamma_{e}$-homothety invariant.

(b) There exists $g \in k_{r}[x]$ such that $f(x)=g\left(x^{\frac{q-1}{e}}\right)$.

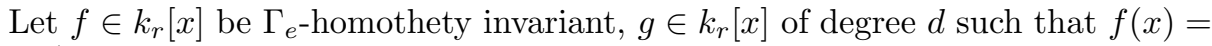
$g\left(x^{\frac{q-1}{e}}\right)$ and $\psi: k \rightarrow \overline{\mathbb{Q}}_{\ell}^{\star}$ a non-trivial additive character. Weil's bound gives in this case

$$
\left|\sum_{x \in k_{r}} \psi\left(\operatorname{Tr}_{k_{r} / k}(f(x))\right)\right| \leq\left(\frac{d(q-1)}{e}-1\right) q^{\frac{r}{2}}
$$

On the other hand,

$$
\begin{gathered}
\sum_{x \in k_{r}} \psi\left(\operatorname{Tr}_{k_{r} / k}(f(x))\right)=\psi\left(\operatorname{Tr}_{k_{r} / k}(f(0))\right)+\sum_{x \in k_{r}^{\star}} \psi\left(\operatorname{Tr}_{k_{r} / k}\left(g\left(x^{\frac{q-1}{e}}\right)\right)\right)= \\
=\psi\left(\operatorname{Tr}_{k_{r} / k}(f(0))\right)+\frac{q-1}{e} \sum_{\mathrm{N}_{k_{r} / k}(x)^{e}=1} \psi\left(\operatorname{Tr}_{k_{r} / k}(g(x))\right)= \\
=\psi\left(\operatorname{Tr}_{k_{r} / k}(f(0))\right)+\frac{q-1}{e} \sum_{\mu^{e}=1} \sum_{\mathrm{N}_{k_{r} / k}(x)=\mu} \psi\left(\operatorname{Tr}_{k_{r} / k}(g(x))\right) .
\end{gathered}
$$

For each $\mu$, we will estimate the sum $\sum_{\mathrm{N}_{k_{r} / k}(x)=\mu} \psi\left(\operatorname{Tr}_{k_{r} / k}(g(x))\right)$ using Weil descent. Fix a basis $\left\{\alpha_{1}, \ldots, \alpha_{r}\right\}$ of $k_{r}$ over $k$, and let $P\left(x_{1}, \ldots, x_{r}\right)=\prod_{\sigma}\left(\sigma\left(\alpha_{1}\right) x_{1}+\right.$ $\left.\cdots+\sigma\left(\alpha_{r}\right) x_{r}\right)$, where the product is taken over all $\sigma \in \operatorname{Gal}\left(k_{r} / k\right)$. Since $P$ is $\operatorname{Gal}\left(k_{r} / k\right)$-invariant, its coefficients are in $k$. By construction, for every $\left(x_{1}, \ldots, x_{r}\right) \in$ $k^{r}$ we have $P\left(x_{1}, \ldots, x_{r}\right)=\mathrm{N}_{k_{r} / k}\left(\alpha_{1} x_{1}+\cdots+\alpha_{r} x_{r}\right)$. Therefore

$$
\begin{gathered}
\sum_{\mathrm{N}_{k_{r} / k}(x)=\mu} \psi\left(\operatorname{Tr}_{k_{r} / k}(g(x))\right)=\sum_{P\left(x_{1}, \ldots, x_{r}\right)=\mu} \psi\left(\operatorname{Tr}_{k_{r} / k}\left(g\left(\alpha_{1} x_{1}+\cdots+\alpha_{r} x_{r}\right)\right)=\right. \\
=\sum_{P\left(x_{1}, \ldots, x_{r}\right)=\mu} \psi\left(\sum_{\sigma} g^{\sigma}\left(\sigma\left(\alpha_{1}\right) x_{1}+\cdots+\sigma\left(\alpha_{r}\right) x_{r}\right)\right)
\end{gathered}
$$

where $g^{\sigma}$ is the polynomial obtained by applying $\sigma$ to the coefficients of $g$, and the sum is taken over all $r$-tuples $\left(x_{1}, \ldots, x_{r}\right) \in k^{r}$ such that $P\left(x_{1}, \ldots, x_{r}\right)=\mu$. By Grothendieck's trace formula, we get

$$
\sum_{\mathrm{N}_{k_{r} / k}(x)=\mu} \psi\left(\operatorname{Tr}_{k_{r} / k}(g(x))\right)=\sum_{i=0}^{2 r-2} \operatorname{Tr}\left(\operatorname{Frob}_{k} \mid \mathrm{H}_{c}^{i}\left(V_{\mu} \otimes \bar{k}, \mathcal{L}_{\psi(G)}\right)\right)
$$

where $V_{\mu}$ is the hypersurface defined in $\mathbb{A}_{k}^{r}$ by the equation $P\left(x_{1}, \ldots, x_{r}\right)=\mu$ and $G=\sum_{\sigma} g^{\sigma}\left(\sigma\left(\alpha_{1}\right) x_{1}+\cdots+\sigma\left(\alpha_{r}\right) x_{r}\right) \in k[x]$ (since it is $\operatorname{Gal}\left(k_{r} / k\right)$-invariant).

Proposition 4.2. Suppose that $g$ has degree d prime to $p$. For any $\mu \in k^{\star}, \mathrm{H}_{c}^{i}\left(V_{\mu} \otimes\right.$ $\left.\bar{k}, \mathcal{L}_{\psi(G)}\right)=0$ for $i \neq r-1$ and $\operatorname{dim} \mathrm{H}_{c}^{r-1}\left(V_{\mu} \otimes \bar{k}, \mathcal{L}_{\psi(G)}\right)=r d^{r-1}$. 
Proof. Over $k_{r}$, the map $\left(x_{1}, \ldots, x_{r}\right) \mapsto\left(\sigma\left(\alpha_{1}\right) x_{1}+\cdots+\sigma\left(\alpha_{r}\right) x_{r}\right)_{\sigma \in \operatorname{Gal}\left(k_{r} / k\right)}$ is a (linear) isomorphism between $\mathbb{A}_{k_{r}}^{r}$ and $\mathbb{A}_{k_{r}}^{\operatorname{Gal}\left(k_{r} / k\right)}$. The pull-back of $P$ under this automorphism is just $x_{1} \cdots x_{r}$. So $V_{\mu} \otimes \bar{k}$ is isomorphic to the hypersurface $x_{1} \cdots x_{r}=\mu$, and the sheaf $\mathcal{L}_{\psi(G)}$ corresponds under this isomorphism to the sheaf $\mathcal{L}_{\psi\left(\sum_{\sigma} g^{\sigma}\left(x_{\sigma}\right)\right)}=\bigotimes_{\sigma} \mathcal{L}_{\psi\left(g^{\sigma}\right)}$ where $\mathcal{L}_{\psi\left(g^{\sigma}\right)}$ is the pull-back of the Artin-Schreier sheaf $\mathcal{L}_{\psi}$ by $g^{\sigma}$.

For every $\sigma \in \operatorname{Gal}\left(k_{r} / k\right)$, the sheaf $\mathcal{L}_{\psi\left(g^{\sigma}\right)}$ is smooth on $\mathbb{A}_{\bar{k}}^{1}$ of rank one, with slope $d$ at infinity. [8, Theorem 5.1] shows that the class of objects of the form $\mathcal{G}[1]$ where $\mathcal{G}$ is a smooth $\overline{\mathbb{Q}}_{\ell}$-sheaf on $\mathbb{G}_{m, \bar{k}}$, tamely ramified at 0 and totally wild at infinity is invariant under convolution. In particular, if $m: \mathbb{G}_{m, \bar{k}}^{\mathrm{Gal}\left(k_{r} / k\right)} \rightarrow \mathbb{G}_{m, \bar{k}}$ is the multiplication map, $\mathrm{R}^{i} m_{!}\left(\bigotimes_{\sigma} \mathcal{L}_{\psi\left(g^{\sigma}\right)}\right)=0$ for $i \neq r-1$ and $\mathrm{R}^{r-1} m_{!}\left(\bigotimes_{\sigma} \mathcal{L}_{\psi\left(g^{\sigma}\right)}\right)$ is smooth on $\mathbb{G}_{m, \bar{k}}$ of rank $r d^{r-1}$, tamely ramified at 0 and totally wild at infinity with Swan conductor $d^{r}$ [ 8 , Theorem 5.1(4,5)]. Taking the fibre at $\mu$ proves the proposition using proper base change.

Corollary 4.3. Suppose that $g$ has degree $d$ prime to $p$. Then

$$
\left|\sum_{x \in k_{r}^{\star}} \psi\left(\operatorname{Tr}_{k_{r} / k}(f(x))\right)\right| \leq r d^{r-1}(q-1) q^{\frac{r-1}{2}}
$$

Proof. Since $\mathcal{L}_{\psi(G)}$ is pure of weight $0, \mathrm{H}_{c}^{r-1}\left(V_{\mu} \otimes \bar{k}, \mathcal{L}_{\psi(G)}\right)$ is mixed of weights $\leq r-1$ for every $\mu$ (in fact it is pure of weight $r-1$ by [8, Theorem 5.1(7)]). So the previous proposition together with (4) implies

$$
\left|\sum_{\mathrm{N}_{k_{r} / k}(x)=\mu} \psi\left(\operatorname{Tr}_{k_{r} / k}(g(x))\right)\right| \leq r d^{r-1} q^{\frac{r-1}{2}}
$$

for every $\mu \in k^{\star}$. We conclude by using (3).

\section{Multiplicative Character sums for homothety inVariant POLYNOMIALS}

Let $e \mid q-1$ an integer and $f(x)=g\left(x^{\frac{q-1}{e}}\right) \in k_{r}[x] \Gamma_{e^{-h o m o t h e t y ~ i n v a r i a n t ~ a s ~ i n ~}}$ the previous section. Let $d=\operatorname{deg}(g)$ and $\chi: k^{\star} \rightarrow \overline{\mathbb{Q}}_{\ell}^{\star}$ a non-trivial multiplicative characer of order $m$. Weil's bound gives

$$
\left|\sum_{x \in k_{r}} \chi\left(\mathrm{N}_{k_{r} / k}(f(x))\right)\right| \leq\left(\frac{d(q-1)}{e}-1\right) q^{\frac{r}{2}}
$$

if $g$ is not an $m$-th power. On the other hand, we have

$$
\begin{gathered}
\sum_{x \in k_{r}} \chi\left(\mathrm{N}_{k_{r} / k}(f(x))\right)=\chi\left(\mathrm{N}_{k_{r} / k}(f(0))\right)+\sum_{x \in k_{r}^{\star}} \chi\left(\mathrm{N}_{k_{r} / k}\left(g\left(x^{\frac{q-1}{e}}\right)\right)\right)= \\
=\chi\left(\mathrm{N}_{k_{r} / k}(f(0))\right)+\frac{q-1}{e} \sum_{\mathrm{N}_{k_{r} / k}(x)^{e}=1} \chi\left(\mathrm{N}_{k_{r} / k}(g(x))\right)= \\
=\chi\left(\mathrm{N}_{k_{r} / k}(f(0))\right)+\frac{q-1}{e} \sum_{\mu^{e}=1} \sum_{\mathrm{N}_{k_{r} / k}(x)=\mu} \chi\left(\mathrm{N}_{k_{r} / k}(g(x))\right) .
\end{gathered}
$$


In order to estimate the sum $\sum_{\mathrm{N}_{k_{r} / k}(x)=\mu} \chi\left(\mathrm{N}_{k_{r} / k}(g(x))\right)$, we may and will assume without loss of generality that $g(0) \neq 0$ : otherwise, writing $g(x)=x^{a} g_{0}(x)$ with $g_{0}(0) \neq 0$,

$$
\begin{gathered}
\sum_{\mathrm{N}_{k_{r} / k}(x)=\mu} \chi\left(\mathrm{N}_{k_{r} / k}(g(x))\right)=\sum_{\mathrm{N}_{k_{r} / k}(x)=\mu} \chi\left(\mathrm{N}_{k_{r} / k}\left(x^{a} g_{0}(x)\right)\right)= \\
=\sum_{\mathrm{N}_{k_{r} / k}(x)=\mu} \chi\left(\mathrm{N}_{k_{r} / k}\left(x^{a}\right)\right) \chi\left(\mathrm{N}_{k_{r} / k}\left(g_{0}(x)\right)\right)=\chi(\mu)^{a} \sum_{\mathrm{N}_{k_{r} / k}(x)=\mu} \chi\left(\mathrm{N}_{k_{r} / k}\left(g_{0}(x)\right)\right),
\end{gathered}
$$

with $\left|\chi(\mu)^{a}\right|=1$.

Let $P=\prod_{\sigma}\left(\sigma\left(\alpha_{1}\right) x_{1}+\cdots+\sigma\left(\alpha_{r}\right) x_{r}\right)$ be as in the previous section, then

$$
\begin{gathered}
\sum_{\mathrm{N}_{k_{r} / k}(x)=\mu} \chi\left(\mathrm{N}_{k_{r} / k}(g(x))\right)=\sum_{P\left(x_{1}, \ldots, x_{r}\right)=\mu} \chi\left(\mathrm{N}_{k_{r} / k}\left(g\left(\alpha_{1} x_{1}+\cdots+\alpha_{r} x_{r}\right)\right)\right)= \\
=\sum_{P\left(x_{1}, \ldots, x_{r}\right)=\mu} \chi\left(\prod_{\sigma} g^{\sigma}\left(\sigma\left(\alpha_{1}\right) x_{1}+\cdots+\sigma\left(\alpha_{r}\right) x_{r}\right)\right)
\end{gathered}
$$

so, by Grothendieck's trace formula,

$$
\sum_{\mathrm{N}_{k_{r} / k}(x)=\mu} \chi\left(\mathrm{N}_{k_{r} / k}(g(x))\right)=\sum_{i=0}^{2 r-2} \operatorname{Tr}\left(\operatorname{Frob}_{k} \mid \mathrm{H}_{c}^{i}\left(V_{\mu} \otimes \bar{k}, \mathcal{L}_{\chi(H)}\right)\right)
$$

where $V_{\mu}$ is the same as in the previous section and $H\left(x_{1}, \ldots, x_{r}\right)=\prod_{\sigma} g^{\sigma}\left(\sigma\left(\alpha_{1}\right) x_{1}+\right.$ $\left.\cdots+\sigma\left(\alpha_{r}\right) x_{r}\right)$, the product taken over the elements of $\operatorname{Gal}\left(k_{r} / k\right)$.

Over $k_{r}$, the map $\left(x_{1}, \ldots, x_{r}\right) \mapsto\left(\sigma\left(\alpha_{1}\right) x_{1}+\cdots+\sigma\left(\alpha_{r}\right) x_{r}\right)_{\sigma \in \operatorname{Gal}\left(k_{r} / k\right)}$ is an isomorphism betweem $\mathbb{A}_{k_{r}}^{r}$ and $\mathbb{A}_{k_{r}}^{\operatorname{Gal}\left(k_{r} / k\right)}$, and the pull-back of $P$ under this automorphism is $x_{1} \cdots x_{r}$. So $V_{\mu} \otimes \bar{k}$ is isomorphic to the hypersurface $x_{1} \cdots x_{r}=\mu$, and the sheaf $\mathcal{L}_{\chi(H)}$ corresponds under this isomorphism to the sheaf $\mathcal{L}_{\chi}\left(\prod_{\sigma} g^{\sigma}\left(x_{\sigma}\right)\right)=$ $\bigotimes_{\sigma} \mathcal{L}_{\chi\left(g^{\sigma}\right)}$ where $\mathcal{L}_{\chi\left(g^{\sigma}\right)}$ is the pull-back of the Kummer sheaf $\mathcal{L}_{\chi}$ by $g^{\sigma}$. Thus $\operatorname{dim} \mathrm{H}_{c}^{i}\left(V_{\mu} \otimes \bar{k}, \mathcal{L}_{\chi(H)}\right)=\operatorname{dim} \mathrm{H}_{c}^{i}\left(\left\{x_{1} \cdots x_{r}=\mu\right\}, \bigotimes_{\sigma} \mathcal{L}_{\chi\left(g^{\sigma}\right)}\right)$. By proper base change, the group $\mathrm{H}_{c}^{i}\left(\left\{x_{1} \cdots x_{r}=\mu\right\}, \bigotimes_{\sigma} \mathcal{L}_{\chi\left(g^{\sigma}\right)}\right)$ is the fibre at $\mu$ of the sheaf $\mathrm{R}^{i} m_{!}\left(\bigotimes_{\sigma} \mathcal{L}_{\chi\left(g^{\sigma}\right)}\right)$, where $m: \mathbb{A}_{\bar{k}}^{\operatorname{Gal}\left(k_{r} / k\right)} \rightarrow \mathbb{A}_{\bar{k}}$ is the multiplication map.

Proposition 5.1. Let $g_{1}, \ldots, g_{r} \in k_{r}[x]$ be square-free of degree $d$ with $g_{i}(0) \neq 0$, $m: \mathbb{A}_{k_{r}}^{r} \rightarrow \mathbb{A}_{k_{r}}^{1}$ the multiplication map and $K_{r}:=\operatorname{R} m_{!}\left(\mathcal{L}_{\chi\left(g_{1}\right)} \otimes \cdots \otimes \mathcal{L}_{\chi\left(g_{r}\right)}\right)$. Suppose that $\chi^{d}$ is not trivial. Then $K_{r}=\mathcal{L}_{r}[1-r]$ for a middle extension sheaf $\mathcal{L}_{r}$ of generic rank $r^{r-1}$ and pure of weight $r-1$ (on the open set where it is smooth), which is totally ramified at infinity and unipotent at 0 , with $\mathrm{H}_{c}^{1}\left(\mathbb{A}_{\bar{k}}, \mathcal{L}_{r}\right)$ pure of weight $r$ and dimension $(d-1)^{r}$.

Proof. We will proceed by induction, as in [1, Théorème 7.8]. For $r=1, \mathcal{L}_{r}=\mathcal{L}_{\chi\left(g_{1}\right)}$ and all results are well known (see e.g. [11]). The sheaf is smooth of rank 1 on the complement of the set of roots of $g_{1}$, and the monodromy group at a root $\alpha$ acts via the non-trivial character $\chi$, so $\mathcal{L}_{\chi\left(g_{1}\right)}$ is a middle extension at $\alpha$.

Suppose everything has been proven for $r-1$. Then

$$
\begin{gathered}
K_{r}=\mathrm{R} m_{!}\left(\mathcal{L}_{\chi\left(g_{1}\right)} \otimes \cdots \otimes \mathcal{L}_{\chi\left(g_{r}\right)}\right)=\mathrm{R} m_{2 !}\left(\mathrm{R} m_{1 !}\left(\mathcal{L}_{\chi\left(g_{1}\right)} \otimes \cdots \otimes \mathcal{L}_{\chi\left(g_{r-1}\right)}\right) \otimes \mathcal{L}_{\chi\left(g_{r}\right)}\right)= \\
=\mathrm{R} m_{2 !}\left(K_{r-1} \otimes \mathcal{L}_{\chi\left(g_{r}\right)}\right)=\mathrm{R} m_{2 !}\left(\mathcal{L}_{r-1}[2-r] \otimes \mathcal{L}_{\chi\left(g_{r}\right)}\right) \\
\text { where } m_{1}: \mathbb{A}_{k_{r}}^{r-1} \rightarrow \mathbb{A}_{k_{r}}^{1} \text { and } m_{2}: \mathbb{A}_{k_{r}}^{2} \rightarrow \mathbb{A}_{k_{r}}^{1} \text { are the multiplication maps. }
\end{gathered}
$$


The fibre of $K_{r}$ at $t \in \bar{k}$ is then $\mathrm{R} \Gamma_{c}\left(\{x y=t\} \subseteq \mathbb{A}_{\bar{k}}^{2}, \mathcal{L}_{r-1} \otimes \mathcal{L}_{\chi\left(g_{r}\right)}\right)[2-r]$. If $t \neq 0,\{x y=t\}$ is isomorphic to $\mathbb{G}_{m}$ via the projection on $x$, so the fibre is $\mathrm{R} \Gamma_{c}\left(\mathbb{G}_{m, \bar{k}}, \mathcal{L}_{r-1} \bigotimes \sigma_{t}^{\star} \mathcal{L}_{\chi\left(g_{r}\right)}\right)[2-r]$, where $\sigma_{t}: \mathbb{G}_{m, \bar{k}} \rightarrow \mathbb{G}_{m, \bar{k}}$ is the involution $x \mapsto t / x$. Since $\mathcal{L}_{r-1}$ is totally ramified at 0 (and unramified at infinity) and $\sigma_{t}^{\star} \mathcal{L}_{\chi\left(g_{r}\right)}$ is unramified at 0 (and totally ramified at infinity), their tensor product is totally ramified at both 0 and infinity. In particular, its $\mathrm{H}_{c}^{2}$ is vanishes. On the other hand, $\mathcal{L}_{r-1}$ and $\mathcal{L}_{\chi\left(g_{r}\right)}$ do not have punctual sections [12, Corollary 6 and Proposition 9], so neither does $\mathcal{L}_{r-1} \otimes \sigma_{t}^{\star} \mathcal{L}_{\chi\left(g_{r}\right)}$ and thus its $\mathrm{H}_{c}^{0}$ vanishes. We conclude that the restriction of $K_{r}$ to $\mathbb{G}_{m}$ is a single sheaf placed in degree $1+(r-2)=r-1$.

The fibre of $K_{r}$ at 0 is $\mathrm{R}_{c}\left(\{x y=0\} \subseteq \mathbb{A}_{\bar{k}}^{2}, \mathcal{L}_{r-1} \otimes \mathcal{L}_{\chi\left(g_{r}\right)}\right)[2-r]$. The group $\mathrm{H}_{c}^{2}\left(\{x y=0\}, \mathcal{L}_{r-1} \otimes \mathcal{L}_{\chi\left(g_{r}\right)}\right)$ vanishes, because so does $\mathrm{H}_{c}^{2}$ of its restriction to $x=0$ (which is a constant times $\mathcal{L}_{\chi\left(g_{r}\right)}$, totally ramified at infinity) and to $y=0$ (which is a constant times $\mathcal{L}_{r-1}$, also totally ramified at infinity). The group $\mathrm{H}_{c}^{0}$ also vanishes, because neither the restiction of $\mathcal{L}_{r-1} \otimes \mathcal{L}_{\chi\left(g_{r}\right)}$ to $x=0$ nor its restriction to $y=0$ have punctual sections. So the stalk of $K_{r}$ at 0 is also concentrated in degree $r-1$.

Once we know $K_{r}$ is a single sheaf $\mathcal{L}_{r}=\mathrm{R}^{r-1} m_{!}\left(\mathcal{L}_{\chi\left(g_{1}\right)} \otimes \cdots \otimes \mathcal{L}_{\chi\left(g_{r}\right)}\right)$, since $\mathrm{H}_{c}^{i}\left(\mathbb{A}_{\bar{k}}, \mathcal{L}_{\chi\left(g_{i}\right)}\right)=0$ for $i \neq 1$ and has dimension $d-1$ and is pure of weight 1 for $i=1$ we get, by Künneth, that $\mathrm{H}_{c}^{i}\left(\mathbb{A}_{\bar{k}}, \mathcal{L}_{r}\right)=0$ for $i \neq 1$ and it has dimension $(d-1)^{r}$ and is pure of weight $r$ for $i=1$. Similarly, since the inverse image of $\mathbb{G}_{m, \bar{k}}$ under the multiplication map is $\mathbb{G}_{m, \bar{k}}^{r}, \mathrm{H}_{c}^{1}\left(\mathbb{G}_{m, \bar{k}}, \mathcal{L}_{r}\right)=0$ for $i \neq 1$ and it has dimension $d^{r}$ for $i=1$. In particular, the rank of $\mathcal{L}_{r}$ at 0 is $\chi\left(\mathbb{A} \frac{1}{\bar{k}}, \mathcal{L}_{r}\right)-\chi\left(\mathbb{G}_{m, \bar{k}}, \mathcal{L}_{r}\right)=d^{r}-(d-1)^{r}$.

Let $t \in \bar{k}$ be a point which is not the product of a ramification point of $\mathcal{L}_{r}$ and a ramification point of $\mathcal{L}_{\chi\left(g_{r}\right)}$. Then at every point of $\mathbb{G}_{m, \bar{k}}$ at least one of $\mathcal{L}_{r-1}$, $\sigma_{t}^{\star} \mathcal{L}_{\chi\left(g_{r}\right)}$ is smooth. Since $\mathcal{L}_{r-1}$ has unipotent monodromy at 0 and $\sigma_{t}^{\star} \mathcal{L}_{\chi\left(g_{r}\right)}$ is unramified at $\infty$, by the Ogg-Shafarevic formula we have

$$
-\chi\left(\mathbb{G}_{m, \bar{k}}, \mathcal{L}_{r-1}\right)=\operatorname{Swan}_{\infty} \mathcal{L}_{r-1}+\sum_{s \in \bar{k}^{\star}}\left(\operatorname{Swan}_{s} \mathcal{L}_{r-1}+\operatorname{drop}_{s} \mathcal{L}_{r-1}\right)
$$

and

$$
-\chi\left(\mathbb{G}_{m, \bar{k}}, \sigma_{t}^{\star} \mathcal{L}_{\chi\left(g_{r}\right)}\right)=\operatorname{Swan}_{0} \mathcal{L}_{\chi\left(g_{r}\right)}+\sum_{s \in \bar{k}^{\star}}\left(\operatorname{Swan}_{t / s} \mathcal{L}_{\chi\left(g_{r}\right)}+\operatorname{drop}_{t / s} \mathcal{L}_{\chi\left(g_{r}\right)}\right)
$$

The local term at $u \in \bar{k}^{\star}$ (sum of the Swan conductor and the drop of the rank) gets multiplied by $e$ upon tensoring with un unramified sheaf of rank $e$. The local term at 0 or $\infty$ (the Swan conductor) gets multiplied by $e$ upon tensoring with a sheaf of rank $e$ with unipotent monodromy. We conclude that

$$
\begin{gathered}
-\chi\left(\mathbb{G}_{m, \bar{k}}, \mathcal{L}_{r-1} \otimes \sigma_{t}^{\star} \mathcal{L}_{\chi\left(g_{r}\right)}\right)=-\left(\operatorname{rank} \mathcal{L}_{\chi\left(g_{r}\right)}\right) \chi\left(\mathbb{G}_{m, \bar{k}}, \mathcal{L}_{r-1}\right)- \\
-\left(\operatorname{rank} \mathcal{L}_{r-1}\right) \chi\left(\mathbb{G}_{m, \bar{k}}, \sigma_{t}^{\star} \mathcal{L}_{\chi\left(g_{r}\right)}\right)=d^{r-1}+d(r-1) d^{r-2}=r d^{r-1} .
\end{gathered}
$$

This is the generic rank of $\mathcal{L}_{r}$

Being a middle extension is a local property which is invariant under tensoring by unramified sheaves. Since, at every point of $\mathbb{G}_{m, \bar{k}}$, at least one of $\mathcal{L}_{r-1}, \sigma_{t}^{\star} \mathcal{L}_{\chi\left(g_{r}\right)}$ is unramified and they are both middle extensions (by the induction hypothesis), their tensor product is a middle extension on $\mathbb{G}_{m, \bar{k}}$. Since it is totally ramified at both 0 and $\infty$, we conclude that $\mathrm{H}_{c}^{1}\left(\mathbb{G}_{m, \bar{k}}, \mathcal{L}_{r-1} \otimes \sigma_{t}^{\star} \mathcal{L}_{\chi\left(g_{r}\right)}\right)$ is pure of weight 
$(r-2)+1=r-1$ [2, Théorème 3.2.3]. So $\mathcal{L}_{r}$ is pure of weight $r-1$ on the open set where it is smooth.

Now let $j_{W}: W \hookrightarrow \mathbb{A}_{\bar{k}}^{1}$ be the inclusion of the largest open sen on which $\mathcal{L}_{r}$ is smooth. Since $\mathcal{L}_{r}$ has no punctual sections, there is an injection $0 \rightarrow \mathcal{L}_{r} \rightarrow$ $j_{W \star} j_{W}^{\star} \mathcal{L}_{r}$, let $\mathcal{Q}$ be its punctual cokernel. We have an exact sequence

$$
0 \rightarrow \mathrm{H}_{c}^{0}\left(\mathbb{A}_{\bar{k}}^{1}, \mathcal{Q}\right) \rightarrow \mathrm{H}_{c}^{1}\left(\mathbb{A}_{\bar{k}}^{1}, \mathcal{L}_{r}\right) \rightarrow \mathrm{H}_{c}^{1}\left(\mathbb{A}_{\bar{k}}^{1}, j_{W \star j_{W}}^{\star} \mathcal{L}_{r}\right) \rightarrow 0
$$

where $\mathrm{H}_{c}^{0}\left(\mathbb{A}_{\bar{k}}, \mathcal{Q}\right)$ has weight $\leq r-1$. Since $\mathrm{H}_{c}^{1}\left(\mathbb{A}_{\bar{k}}, \mathcal{L}_{r}\right)$ is pure of weight $r$, we conclude that $\mathrm{H}_{c}^{0}\left(\mathbb{A}_{\bar{k}}, \mathcal{Q}\right)$ and therefore $\mathcal{Q}$ are zero, so $\mathcal{L}_{r}$ is a middle extension. Now let $j: \mathbb{A}_{\bar{k}} \hookrightarrow \mathbb{P}_{\bar{k}}^{\frac{1}{k}}$ be the inclusion, again we get an exact sequence

$$
0 \rightarrow \mathcal{L}_{r}^{I_{\infty}} \rightarrow \mathrm{H}_{c}^{1}\left(\mathbb{A}_{\bar{k}}^{1}, \mathcal{L}_{r}\right) \rightarrow \mathrm{H}_{c}^{1}\left(\mathbb{P}_{\bar{k}}^{1}, j_{\star} \mathcal{L}_{r}\right) \rightarrow 0
$$

with $\mathcal{L}_{r}^{I_{\infty}}$ of weight $\leq r-1$, since $\mathrm{H}_{c}^{1}\left(\mathbb{A}_{\bar{k}}^{1}, \mathcal{L}_{r}\right)$ is pure of weight $r$ we conclude that $\mathcal{L}_{r}^{I_{\infty}}=0$, that is, $\mathcal{L}_{r}$ is totally ramified at infinity.

It remains to prove that $\mathcal{L}_{r}$ has unipotent monodromy at zero. Consider the exact sequence

$$
0 \rightarrow \mathcal{L}_{r}^{I_{0}} \rightarrow \mathrm{H}_{c}^{1}\left(\mathbb{G}_{m, \bar{k}}, \mathcal{L}_{r}\right) \rightarrow \mathrm{H}_{c}^{1}\left(\mathbb{A}_{\bar{k}}^{1}, \mathcal{L}_{r}\right) \rightarrow 0
$$

which identifies $\mathcal{L}_{r}^{I_{0}}$ with the weight $<r$ part of $\mathrm{H}_{c}^{1}\left(\mathbb{G}_{m, \bar{k}}, \mathcal{L}_{r}\right)$. Since $\mathrm{H}_{c}^{1}\left(\mathbb{G}_{m, \bar{k}}, \mathcal{L}_{r}\right)=$ $\bigotimes_{i=1}^{r} \mathrm{H}_{c}^{1}\left(\mathbb{G}_{m, \bar{k}}, \mathcal{L}_{\chi\left(g_{i}\right)}\right)$ and $\mathrm{H}_{c}^{1}\left(\mathbb{G}_{m, \bar{k}}, \mathcal{L}_{\chi\left(g_{i}\right)}\right)$ has $d-1$ Frobenius eigenvalues of weight 1 and one of weight 0 , we conclude that $\mathrm{H}_{c}^{1}\left(\mathbb{G}_{m, \bar{k}}, \mathcal{L}_{r}\right)$ has $\left(\begin{array}{c}r \\ i\end{array}\right)(d-1)^{i}$ eigenvalues of weight $i$ for every $i=0, \ldots, r$. By [8, Theorem 7.0.7], an eigenvalue of weight $i<r$ on $\mathcal{L}_{r}^{I_{0}}$ corresponds to a unipotent Jordan block of size $r-i$ for the action of $I_{0}$. So the sum of the sizes of the unipotent Jordan blocks for the monodromy of $\mathcal{L}_{r}$ at 0 is

$$
\begin{gathered}
\sum_{i=0}^{r-1}\left(\begin{array}{c}
r \\
i
\end{array}\right)(d-1)^{i}(r-i)=r \sum_{i=0}^{r-1}\left(\begin{array}{c}
r \\
i
\end{array}\right)(d-1)^{i}-r \sum_{i=0}^{r-1}\left(\begin{array}{c}
r-1 \\
i-1
\end{array}\right)(d-1)^{i}= \\
=r \sum_{i=0}^{r-1}\left(\begin{array}{c}
r-1 \\
i
\end{array}\right)(d-1)^{i}=r(1+d-1)^{r-1}=r d^{r-1}
\end{gathered}
$$

which is the generic rank of $\mathcal{L}_{r}$. So the unipotent Jordan blocks fill out the entire monodromy at 0 .

Corollary 5.2. Suppose that $g$ is square-free of degree $d$ prime to $p$ and $\chi^{d}$ is not trivial. For any $\mu \in k^{\star}, \mathrm{H}_{c}^{i}\left(V_{\mu} \otimes \bar{k}, \mathcal{L}_{\chi(H)}\right)=0$ for $i \neq r-1$ and $\operatorname{dim}_{c}^{r-1}\left(V_{\mu} \otimes\right.$ $\left.\bar{k}, \mathcal{L}_{\chi(H)}\right)=r d^{r-1}$.

Proof. Apply the previous proposition with $\left(g_{1}, \ldots, g_{r}\right)=\left(g^{\sigma}\right)_{\sigma \in \operatorname{Gal}\left(k_{r} / k\right)}$, and proper base change.

Corollary 5.3. Suppose that $g$ is square-free of degree $d$ prime to $p$ and $\chi^{d}$ is not trivial. Then

$$
\left|\sum_{x \in k_{r}^{\star}} \chi\left(\mathrm{N}_{k_{r} / k}(f(x))\right)\right| \leq r d^{r-1}(q-1) q^{\frac{r-1}{2}}
$$


Proof. Since $\mathcal{L}_{\chi(H)}$ is pure of weight $0, \mathrm{H}_{c}^{r-1}\left(V_{\mu} \otimes \bar{k}, \mathcal{L}_{\chi(H)}\right)$ has weights $\leq r-1$ for every $\mu$. So the previous corollary together with (6) implies

$$
\left|\sum_{\mathrm{N}_{k_{r} / k}(x)=\mu} \chi\left(\mathrm{N}_{k_{r} / k}(g(x))\right)\right| \leq r d^{r-1} q^{\frac{r-1}{2}}
$$

for every $\mu \in k^{\star}$. We conclude by using (5).

Remark 5.4. The following example shows that the hypothesis $\chi^{d}$ non-trivial is necessary. Let $p$ be odd, $r=2, g(x)=x^{2}+1$ and $\rho: k^{\star} \rightarrow \overline{\mathbb{Q}}_{\ell}^{\star}$ the quadratic character. Then

$$
\begin{gathered}
\sum_{\mathrm{N}_{k_{r} / k}(x)=1} \rho\left(\mathrm{N}_{k_{r} / k}\left(x^{2}+1\right)\right)=\sum_{x^{q+1}=1} \rho\left(\left(x^{2}+1\right)\left(x^{2 q}+1\right)\right)= \\
=\sum_{x^{q+1}=1} \rho\left(x^{2}+x^{2 q}+2\right)=\sum_{x^{q+1}=1} \rho\left(\left(x+x^{q}\right)^{2}\right) \geq q-1
\end{gathered}
$$

since $x+x^{q}=\operatorname{Tr}_{k_{r} / k}(x) \in k$ and therefore $\rho\left(\left(x+x^{q}\right)^{2}\right)=\rho\left(x+x^{q}\right)^{2}=1$ unless $x+x^{q}=0$, which only happens for $x^{2}=-1$, that is, for at most two values of $x$. So we can never have an estimate of the form

$$
\left|\sum_{\mathrm{N}_{k_{r} / k}(x)=1} \rho\left(\mathrm{N}_{k_{r} / k}\left(x^{2}+1\right)\right)\right| \leq C \cdot q^{\frac{1}{2}}
$$

which is valid for all $q$.

\section{REFERENCES}

1. P. Deligne, Application de la formule des traces aux sommes trigonométriques dans Cohomologie Etale, Séminaire de Géométrie Algébrique du Bois-Marie, SGA 4 1/2, Lecture Notes in Math 569, 168-232.

2. _ La conjecture de Weil. II, Publications Mathématiques de l'IHÉS 52 (1980), no. 1, $137-252$.

3. L. Fu, Calculation of l-adic local Fourier transformations, arXiv:math/0702436 (2007).

4. L. Fu and D. Wan, Moment L-functions, partial L-functions and partial exponential sums, Mathematische Annalen 328 (2004), no. 1, 193-228.

5. C. Douglas Haessig and Antonio Rojas-Leon, L-functions of symmetric powers of the generalized Airy family of exponential sums, arXiv:1008.0408 (2010).

6. H. Hasse, Theorie der relativ-zyklischen algebraischen Funktionenkörper, insbesondere bei endlichem Konstantenkörper., Journal für die reine und angewandte Mathematik (1935), no. $172,37-54$.

7. N.M. Katz, On the Monodromy Groups Attached to Certain Families of Exponential Sums, Duke Mathematical Journal 54 (1987).

8. __ Gauss Sums, Kloosterman Sums, and Monodromy Groups, Annals of Mathematics Studies, vol. 116, Princeton University Press, 1988.

9. $\_$Exponential Sums and Differential Equations, Annals of Mathematics Studies, vol. 124, Princeton University Press, 1990.

10. nite Fields and Their Applications 7 (2001), no. 1, 45-69.

11. _ Estimates for nonsingular multiplicative character sums, International Mathematics Research Notices (2002), no. 7, 333-349.

12. _ A semicontinuity result for monodromy under degeneration, Forum Mathematicum 15 (2003), no. 2, 191-200.

13. N.M. Katz and G. Laumon, Transformation de Fourier et majoration de sommes exponentielles, Publications Mathématiques de l'IHÉS 62 (1985), no. 1, 145-202. 
14. G. Laumon, Transformation De Fourier, Constantes d'Equations Fonctionnelles Et Conjecture De Weil, Publications Mathématiques de l'IHÉS 65 (1987), no. 1, 131-210.

15. Antonio Rojas-Leon and Daqing Wan, Big improvements of the Weil bound for Artin-Schreier curves, arXiv:1004.2224 (2010).

16. O. Šuch, Monodromy of Airy and Kloosterman sheaves, Duke Mathematical Journal 103 (2000), no. 3, 397-444.

17. A. Weil, On some exponential sums, Proceedings of the National Academy of Sciences of the United States of America 34 (1948), no. 5, 204.

Departamanto de Álgebra, Universidad de Sevilla, Apdo 1160, 41080 Sevilla, Spain

E-MAIL: AROJAS@US.ES 